\title{
Fermionic vacuum currents in topologically nontrivial braneworlds: Two-brane geometry
}

\author{
S. Bellucci®,$^{1, *}$ A. A. Saharian $\oplus^{2, \dagger}$ H. G. Sargsyan $\oplus^{2, \ddagger}$ and V. V. Vardanyan ${ }^{2,3, \S}$ \\ ${ }^{1}$ INFN, Laboratori Nazionali di Frascati, Via Enrico Fermi 40, 00044 Frascati, Italy \\ ${ }^{2}$ Department of Physics, Yerevan State University, 1 Alex Manoogian Street, 0025 Yerevan, Armenia \\ ${ }^{3}$ Lorentz Institute for Theoretical Physics, Leiden University, 2333 CA Leiden, Netherlands
}

(Received 12 August 2019; accepted 28 January 2020; published 27 February 2020)

\begin{abstract}
The vacuum expectation value (VEV) of the fermionic current density is investigated in the geometry of two parallel branes in locally AdS spacetime with a part of spatial dimensions compactified to a torus. Along the toral dimensions quasiperiodicity conditions are imposed with general phases and the presence of a constant gauge field is assumed. The influence of the latter on the VEV is of the Aharonov-Bohm type. Different types of boundary conditions are discussed on the branes, including the bag boundary condition and the conditions arising in $Z_{2}$-symmetric braneworld models. Nonzero vacuum currents appear along the compact dimensions only. In the region between the branes they are decomposed into the brane-free and brane-induced contributions. Both these contributions are periodic functions of the magnetic flux enclosed by compact dimensions with the period equal to the flux quantum. Depending on the boundary conditions, the presence of the branes can either increase or decrease the vacuum current density. For a part of boundary conditions, a memory effect is present in the limit when one of the branes tends to the AdS boundary. Unlike to the fermion condensate and the VEV of the energy-momentum tensor, the VEV of the current density is finite on the branes. Applications are given to higher-dimensional generalizations of the RandallSundrum models with two branes and with toroidally compact subspace. The features of the fermionic current are discussed in odd-dimensional parity and time-reversal symmetric models. The corresponding results for three-dimensional spacetime are applied to finite length curved graphene tubes threaded by a magnetic flux. It is shown that a nonzero current density can also appear in the absence of the magnetic flux if the fields corresponding to two different points of the Brillouin zone obey different boundary conditions on the tube edges.
\end{abstract}

DOI: 10.1103/PhysRevD.101.045020

\section{INTRODUCTION}

In quantum field theory the vacuum is defined as a state of quantum fields with the zero number of quanta. It depends on the choice of the complete set of mode functions in terms of which the quantization of fields is done (see, for instance, [1]). The mode functions and, as a consequence of that, the properties of the vacuum are sensitive to both the local and global characteristics of the background geometry. In particular, the vacuum expectation values (VEVs) of physical observables depend on the

\footnotetext{
bellucci@lnf.infn.it

†saharian@ysu.am

hayk28.sargsyan@yahoo.com

§vardanyan@lorentz.leidenuniv.nl
}

Published by the American Physical Society under the terms of the Creative Commons Attribution 4.0 International license. Further distribution of this work must maintain attribution to the author(s) and the published article's title, journal citation, and DOI. Funded by SCOAP. boundary conditions imposed on fields. This dependence is manifested in the Casimir effect [2] and has been investigated for large number of bulk and boundary geometries. The boundary conditions may have different physical origins. They can be induced by the presence of boundaries (material boundaries in quantum electrodynamics, domain walls separating different phases, horizons in gravitational physics, branes in braneworld scenarios) or as a consequence of nontrivial spatial topology. In some models formulated in background of manifolds with edges, the imposition of boundary conditions on those edges is required to prevent the unitarity of the theory. In the present paper we consider a physical problem with different sources for the polarization of vacuum. They include the background gravitational field, gauge field, boundaries, and nontrivial spatial topology.

The background geometry we are going to discuss is locally anti-de Sitter (AdS) one. Being the maximally symmetric solution of the vacuum Einstein equations with a negative cosmological constant, AdS spacetime is among the most popular geometries in quantum field theory on 
curved backgrounds. First of all, because of high symmetry, a large number of physical problems are exactly solvable on that background. These solutions may help to shed light on the influence of gravitational field on quantum matter in less symmetric geometries. The lack of global hyperbolicity and the presence of the modes regular and irregular on the AdS boundary give rise to new principal questions in the quantization procedure of fields having no analogs in quantum field theory on the Minkowski bulk. The importance of the corresponding investigations is also related to the fact that the AdS spacetime naturally appears as a ground state in extended supergravity and in string theories and also as the near horizon geometry of the extremal black holes and domain walls.

The further increase of the interest to the AdS based field theories is motivated by a crucial role of the corresponding geometry in two exciting developments of theoretical physics in the past decade. The first one is the braneworld scenario with large extra dimensions which provides a geometrical solution to the hierarchy problem between the gravitational and electroweak energy scales [3]. The corresponding models are usually formulated on higherdimensional AdS bulk with branes parallel to the AdS boundary and the weak coupling of 4-dimensional gravity is generated by the large physical volume of extra dimensions. Braneworlds naturally appear in the string/M theory context and present a novel setting for the discussion of phenomenological and cosmological issues related to extra dimensions. The second development is related to the AdS/CFT correspondence (for reviews see [4]) that relates string theories or supergravity in the AdS bulk with a conformal field theory living on its boundary. This duality between two different theories has many interesting consequences and provides a powerful tool for the investigation of gauge field theories. Among the recent developments of the AdS/CFT correspondence is the application to strongcoupling problems in condensed matter physics (familiar examples include holographic superconductors, quantum phase transitions, and topological insulators) [5].

In the present paper the global properties of the background geometry will be different from those for AdS spacetime. It will be assumed that a part of the Poincaré coordinates in the AdS line element are compactified on a torus. In addition, we assume the presence of two branes parallel to the AdS boundary. As a consequence, two types of conditions are imposed on the operators of quantum fields: periodicity conditions along compact dimensions and boundary conditions on the branes. In the RandallSundrum type branewrolds the latter are dictated by the $Z_{2}$-symmetry with respect to the branes. Both these conditions modify the spectrum of vacuum fluctuations and give rise to the Casimir type contributions in the physical characteristics of the vacuum state. In particular, motivated by the problem of radion stabilization in braneworld scenario, the brane-induced quantum effects have been intensively investigated for scalar [6], fermionic [7-10] and vector fields [11]. The models with de Sitter branes have been considered in [12]. The Casimir effect in AdS spacetime with additional compact subspaces is discussed in [13]. The expectation value of the surface energy-momentum tensor for a scalar field, induced on branes, and related cosmological constant are studied in [14].

The papers cited above consider mainly the vacuum energy or the VEV of the energy-momentum tensor. For charged fields, an important local characteristics are the expectation values of the charge and current densities. For scalar and fermionic fields in flat backgrounds with a part of spatial dimensions compactified to a torus, these expectation values at zero and finite temperatures were considered in Refs. [15-17]. The results for fermionic fields in a special case of two spatial dimensions have been applied to cylindrical and toroidal carbon nanotubes described in terms of the long-wavelength effective Dirac model. The boundary-induced effects of the Casimir type on the vacuum charges and currents are discussed in $[18,19]$. The fermionic current density induced by a magnetic flux in planar rings with concentric circular boundaries has been investigated in [20]. The persistent currents in normal metal rings having a similar physical origin have been experimentally observed in [21]. The effects of edges on the fermion condensate and the currents in two-dimensional conical spaces are discussed in [22]. More complicated problems for the vacuum currents in locally de Sitter and AdS background geometries with toroidally compactified spatial dimensions are considered in [23] and [24,25]. Induced current in AdS spacetime in the presence of a cosmic string and compactified spatial dimension is studied in [26]. The brane-induced effects on the current density for a charged scalar field with Robin boundary conditions in locally AdS bulk are investigated in [27,28]. The corresponding problem for a fermionic field in the geometry of a single brane with bag boundary condition has been considered in [29]. Continuing in this line of investigations, here we consider the fermionic vacuum currents for two-brane geometry in background of locally AdS spacetime with compact dimensions and for different combinations of the boundary conditions on them.

The organization of the paper is as follows. In the next section we specify the bulk and boundary geometries, the topology and the boundary conditions imposed on the field. In Sec. III, a complete set of the positive and negative energy solutions to the Dirac equation is presented in the region between two branes and the eigenvalues of the radial quantum number are specified. The VEV of the current density for the bag boundary condition on the branes is investigated in Sec. IV. Two alternative representations are provided and the asymptotic behavior is discussed in various limiting regions of the parameters. In Sec. V we consider the VEV of the current density for another type of boundary condition that differs from the bag boundary 
condition by the sign of the term containing the normal to the boundary. In Sec. VI, the fermionic current in $Z_{2}$-symmetric braneworld models with two branes is investigated. Various combinations of the boundary conditions, dictated by the $Z_{2}$-symmetry with respect to the branes, are discussed. The features of the fermionic current in odd-dimensional parity and time-reversal symmetric models are considered in Sec. VII and applications are given to the Dirac model describing the long wavelength properties of curved graphene tubes. The main results are summarized in Sec. VIII. In Appendix, by using the generalized Abel-Plana formula, a summation formula is derived for series over the eigenmodes of the fermionic field in the region between the branes for boundary conditions on the field operator discussed in the main text.

\section{PROBLEM SETUP}

In this section we describe the bulk and boundary geometries, the field and the periodicity and boundary conditions.

\section{A. Background geometry}

Consider a $(D+1)$-dimensional spacetime with the line element

$d s^{2}=e^{-2 y / a} \eta_{i k} d x^{i} d x^{k}-d y^{2}=(a / z)^{2} \eta_{\mu \nu} d x^{\mu} d x^{\nu}$,

where $a$ is a constant having the dimension of length, the Latin and Greek indices run over $0,1, \ldots, D-1$ and $0,1, \ldots, D$, respectively, $\eta_{\mu \nu}=\operatorname{diag}(1,-1, \ldots,-1)$ is the metric tensor for the Minkowski spacetime in the Cartesian coordinates. The conformal coordinate $z=x^{D}$ is expressed in terms of the coordinate $y,-\infty<y<+\infty$, by the relation $z=a e^{y / a}$ with the range $0 \leq z<\infty$. The line element (2.1) coincides with the one for the $(D+1)$ dimensional AdS spacetime, described in Poincaré coordinates. In the case of AdS spacetime, for the coordinates $x^{i}, i=1, \ldots, D$, one has $-\infty<x^{i}<+\infty$. The global properties of the geometry we are going to consider here will be different. Namely, we assume that the subspace with the coordinates $\mathbf{x}_{(q)}=\left(x^{p+1}, \ldots, x^{D-1}\right), q=D-p-1$, is compactified to a $q$-dimensional torus $T^{q}=\left(S^{1}\right)^{q}$ with the lengths of the compact dimensions $L_{l}, 0 \leq x^{l} \leq L_{l}$, $l=p+1, \ldots, D-1$. In what follows we will denote by $V_{q}=L_{p+1} \cdots L_{D-1}$ the volume of the compact subspace. For the coordinates $\mathbf{x}_{(p)}=\left(x^{1}, \ldots, x^{p}\right)$, as usual, one has $-\infty<x^{i}<+\infty, i=1, \ldots, p$, and, hence, the subspace covered by the set of coordinates $\left(\mathbf{x}_{(p)}, \mathbf{x}_{(q)}\right)=$ $\left(x^{1}, \ldots, x^{D-1}\right)$ has topology $R^{p} \times T^{q}$. Note that the constant $L_{l}$ is the coordinate length of the $l$ th compact dimension. The physical (or proper) length $L_{(p) l}$ of that dimension, measured by an observer having a fixed $z$ coordinate, is given by $L_{(p) l}=(a / z) L_{l}$ and it decreases with increasing $z$ (see Fig. 5 below for the $D=2$ spatial geometry embedded in a three-dimensional Euclidean space).

The last relation in (2.1) shows that the geometry under consideration is conformally related to the half (with $\left.0 \leq x^{D}<\infty\right)$ of the locally Minkowskian $(D+1)$ dimensional spacetime with spatial topology $R^{p+1} \times T^{q}$. The Minkowskian counterpart contains a boundary $x^{D}=0$ the boundary condition on which is determined by the boundary condition imposed on the AdS boundary $z=0$. The AdS horizon is presented by the hypersurface $z=\infty$. The toroidal compactification under consideration does not change the local geometry and the Ricci tensor $R_{\mu}^{\nu}=$ $-D \delta_{\mu}^{\nu} / a^{2}$ is the same as that for AdS spacetime.

As a boundary geometry we will assume the presence of two codimension one branes located at $y=y_{1}$ and $y=y_{2}$, $y_{1}<y_{2}$. For the corresponding values of the conformal coordinate $z$ one has $z_{j}=a e^{y_{j} / a}, j=1,2$. Note that the physical distance between the branes is given by $y_{2}-y_{1}=$ $a \ln \left(z_{2} / z_{1}\right)$ and they have spatial topology $R^{p} \times T^{q}$. For the extrinsic curvature tensor of the brane at $z=z_{j}$ one has $K_{i k}^{(j)}= \pm g_{i k} / a$, where the upper and lower signs correspond to the regions $z \leq z_{j}$ and $z \geq z_{j}$. As a consequence of the nonzero extrinsic curvature, the physical effects of the brane on the properties of the quantum vacuum are different in those regions. In the generalized Randall-Sundrum type models with additional compact dimensions, the hypersurfaces $y=y_{1}$ and $y=y_{2}$ correspond to the hidden and visible branes, respectively. Higher dimensional generalizations of the braneworld models with compact dimensions are, in particular, important from the viewpoint of underlying fundamental theories in higher dimensions such as superstring/ $\mathrm{M}$ theories. The consideration of more general spacetimes may provide interesting extensions of the Randall-Sundrum mechanism for the geometric origin of the hierarchy.

The term "brane" for the boundary, used in the paper, is in some sense, conditional. The role of the branes is just to impose boundary conditions on a quantum field and the braneworlds are one of the motivations for the problem. The boundary conditions on fermionic fields imposed by the branes in $Z_{2}$-symmetric models of Randall-Sundrum type and the corresponding current densities will be discussed in Sec. VI. However, the boundary conditions on quantum fields may rise by other physical reasons and the applications of the results given below are wider. As another example, in Sec. VII we consider an application to curved graphene tubes described by an effective Dirac model. In that example the boundaries correspond to the edges of the tube.

\section{B. Field and boundary conditions}

Having specified the bulk and boundary geometries, now we pass to the field content. We consider a charged fermionic field $\psi(x)$ with the mass parameter $m$ in the 
presence of an external classical Abelian gauge field $A_{\mu}(x)$. Here and in what follows the shorthand notation $x=\left(x^{0}=\right.$ $\left.t, x^{1}, \ldots, x^{D}\right)$ is used for the spacetime coordinates. The coupling parameter between the fermionic and gauge fields will be denoted by $e$. For a fermionic field realizing the irreducible representation of the Clifford algebra the number of components of the spinor $\psi(x)$ is equal to $N=2^{[(D+1) / 2]}$, where the square brackets mean the integer part. Introducing the gauge extended covariant derivative operator $D_{\mu}=\partial_{\mu}+\Gamma_{\mu}+i e A_{\mu}$, with $\Gamma_{\mu}$ being the spin connection, the field equation is written as

$$
\left(i \gamma^{\mu} D_{\mu}-m\right) \psi(x)=0 .
$$

The curved spacetime $N \times N$ Dirac matrices are expressed in terms of the corresponding flat spacetime matrices $\gamma^{(b)}$ by the relation $\gamma^{\mu}=e_{(b)}^{\mu} \gamma^{(b)}$, where $e_{(b)}^{\mu}$ are the vierbein fields. In the conformal coordinates $x^{\mu}$, with $x^{D}=z$, the vierbein fields can be taken in the form $e_{(b)}^{\mu}=(z / a) \delta_{b}^{\mu}$. With this choice, for the components of the spin connection one gets $\Gamma_{D}=0$ and $\Gamma_{k}=\eta_{k l} \gamma^{(D)} \gamma^{(l)} /(2 z)$ for $k=0, \ldots, D-1$. The field equation (2.2) is invariant under the gauge transformation $\psi(x)=\psi^{\prime}(x) e^{-i e \chi(x)}, A_{\mu}(x)=$ $A_{\mu}^{\prime}(x)+\partial_{\mu} \chi(x)$.

The background geometry is not simply connected and, in addition to the field equation, the periodicity conditions on the field operator should be specified along compact dimensions for the theory to be defined. For the $l$ th compact dimension we will impose the condition

$\psi\left(t, \mathbf{x}_{(p)}, \mathbf{x}_{(q)}+L_{l} \mathbf{e}_{(l)}, x^{D}\right)=e^{i \alpha_{l}} \psi\left(t, \mathbf{x}_{(p)}, \mathbf{x}_{(q)}, x^{D}\right)$,

where $\mathbf{e}_{(l)}$ is the unit vector along the dimension $x^{l}$ with the components $e_{(l)}^{i}=\delta_{l}^{i}$ and $\alpha_{l}, l=p+1, \ldots, D-1$, are constant phases. The special cases, most frequently considered in the literature, correspond to untwisted $\left(\alpha_{l}=0\right)$ and twisted $\left(\alpha_{l}=\pi\right)$ fields. The periodicity conditions with $\alpha_{l} \neq 0$ have been used in the literature to exclude the zero mode of the fermionic field. For the gauge field the simplest configuration will be assumed with $A_{\mu}=$ const. Only the components $A_{l}, l=p+1, \ldots, D-1$, along compact dimensions are physically relevant. Their effects on physical observables are of the Aharonov-Bohm type and they are induced by the nontrivial topology of the background geometry. By the gauge transformation $\left\{\psi(x), A_{\mu}\right\} \rightarrow\left\{\psi^{\prime}(x), A_{\mu}^{\prime}\right\}$, with the transformation function $\chi=b_{\mu} x^{\mu}$ and constant $b_{\mu}$, one gets a new set of fields $\left\{\psi^{\prime}(x), A_{\mu}^{\prime}\right\}=\left\{\psi(x) e^{i e b_{\mu} x^{\prime}}, A_{\mu}-b_{\mu}\right\}$. The periodicity conditions for the field $\psi^{\prime}(x)$ are of the form (2.3) with new phases $\alpha_{l}^{\prime}=\alpha_{l}+e b_{l} L_{l}$. Hence, by the gauge transformation the set of parameters $\left\{\alpha_{l}, A_{l}\right\}$ is transformed to a new set $\left\{\alpha_{l}^{\prime}, A_{l}^{\prime}\right\}=\left\{\alpha_{l}+e b_{l} L_{l}, A_{l}-b_{l}\right\}$. In what follows, it is convenient for us to work in the gauge with $b_{\mu}=A_{\mu}$ with the zero vector potential $A_{\mu}^{\prime}$. The corresponding phases in the periodicity conditions for the field operator $\psi^{\prime}(x)$ (in the following we will omit the primes) will be denoted by $\tilde{\alpha}_{l}$ :

$$
\tilde{\alpha}_{l}=\alpha_{l}+e A_{l} L_{l} .
$$

This shows that the physics depends on the parameters $\alpha_{l}$ and $A_{l}$ in the form of the combination (2.4). The phase shift induced by the vector potential can be presented as $e A_{l} L_{l}=-e A^{l} L_{l}=-2 \pi \Phi_{l} / \Phi_{0}$, where $\Phi_{l}$ is formally interpreted in terms of the magnetic flux enclosed by the $l$ th compact dimension and $\Phi_{0}=2 \pi / e$ is the flux quantum.

In the presence of the branes at $z=z_{j}, j=1,2$, for the theory to be defined one needs to specify the boundary conditions on them. In this section we will assume that the field operator obeys the bag boundary conditions

$$
\left(1+i \gamma^{\mu} n_{\mu}^{(j)}\right) \psi(x)=0, \quad z=z_{j},
$$

with $n_{\mu}^{(j)}$ being the inward pointing normal (with respect to the region under consideration) to the brane at $z=z_{j}$. Other types of boundary conditions on the branes will be discussed in the following sections. The branes divide the background space into three regions: $0 \leq z \leq z_{1}$, $z_{1} \leq z \leq z_{2}$, and $z \geq z_{2}$. The current densities in the regions $0 \leq z \leq z_{1}$ and $z \geq z_{2}$ are the same as those for a single brane located at $z=z_{1}$ and $z=z_{2}$, respectively, and they are investigated in [29]. Here we will be mainly concerned with the region between the branes, $z_{1} \leq z \leq z_{2}$. For that region in (2.5) one has $n_{\mu}^{(j)}=(-1)^{j} \delta_{\mu}^{D} a / z_{j}$.

\section{FERMIONIC MODES IN THE REGION BETWEEN THE BRANES}

In this section we consider a complete set of positive and negative energy modes $\left\{\psi_{\beta}^{(+)}, \psi_{\beta}^{(-)}\right\}$for the fermionic field $\psi(x)$. The collective set $\beta$ of quantum numbers will be specified below. In order to solve the field equation (2.2) we need to choose the representation of the Dirac matrices. As it already has been discussed in [29], it is convenient to take the flat spacetime gamma matrices in the representation

$\gamma^{(b)}=\left(\begin{array}{cc}0 & \chi_{b} \\ (-1)^{1-\delta_{b}^{0}} \chi_{b}^{\dagger} & 0\end{array}\right), \quad b=0,1, \ldots, D-1$,

and $\gamma^{(D)}=s i \operatorname{diag}(1,-1)$ with $s= \pm 1$. In odd-dimensional spacetimes there exist two inequivalent irreducible representations of the Clifford algebra and the values $s=+1$ and $s=-1$ correspond to those representations. In even spacetime dimensions the irreducible representation of the Clifford algebra is unique, up to a similarity transformation, and we can put $s=1$. For $D=2$ we can take $\chi_{0}=$ $\chi_{1}=1$ and the matrices $\gamma^{(b)}$ are expressed in terms of the Pauli matrices $\sigma_{\mathrm{P} \mu}$ as $\gamma^{(0)}=\sigma_{\mathrm{P} 1}, \gamma^{(1)}=i \sigma_{\mathrm{P} 2}, \gamma^{(2)}=s i \sigma_{\mathrm{P} 3}$. 
The commutation relations for the $N / 2 \times N / 2$ matrices $\chi_{b}$ are obtained from those for the Dirac matrices $\gamma^{(b)}$. They are reduced to $\chi_{0}^{\dagger} \chi_{0}=1, \chi_{0} \chi_{b}^{\dagger}=\chi_{b} \chi_{0}^{\dagger}, \chi_{0}^{\dagger} \chi_{b}=\chi_{b}^{\dagger} \chi_{0}$, and

$$
\begin{aligned}
& \chi_{b} \chi_{c}^{\dagger}+\chi_{c} \chi_{b}^{\dagger}=2 \delta_{b c}, \\
& \chi_{b}^{\dagger} \chi_{c}+\chi_{c}^{\dagger} \chi_{b}=2 \delta_{b c},
\end{aligned}
$$

where $b, c=1,2, \ldots, D-1$.

With the curved spacetime gamma matrices $\gamma^{\mu}=$ $(z / a) \delta_{b}^{\mu} \gamma^{(b)}$, the complete set of solutions of the field equation (2.2) (with $A_{\mu}=0$ in the gauge under consideration) can be found in a way similar to that given in [29]. Introducing the one-column matrices $w^{(\sigma)}, \sigma=1, \ldots, N / 2$, with $N / 2$ rows and with the elements $w_{l}^{(\sigma)}=\delta_{l \sigma}$, for the positive and negative energy mode functions one gets

$$
\begin{aligned}
& \psi_{\beta}^{(+)}(x)=z^{\frac{D+1}{2}} e^{i \mathbf{k x}-i \omega t}\left(\begin{array}{c}
\frac{\mathbf{k} \chi \chi_{0}^{\dagger}+i \lambda-\omega}{\omega} Z_{m a+s / 2}(\lambda z) w^{(\sigma)} \\
i \chi_{0}^{\dagger} \frac{\mathbf{k} \chi \chi_{0}^{\dagger}+i \lambda+\omega}{\omega} Z_{m a-s / 2}(\lambda z) w^{(\sigma)}
\end{array}\right), \\
& \psi_{\beta}^{(-)}(x)=z^{\frac{D+1}{2}} e^{i \mathbf{k} \mathbf{x}+i \omega t}\left(\begin{array}{c}
i \chi_{0} \frac{\mathbf{k} \chi^{\dagger} \chi_{0}-i \lambda+\omega}{\omega} Z_{m a+s / 2}(\lambda z) w^{(\sigma)} \\
\frac{\mathbf{k} \chi^{\dagger} \chi_{0}-i \lambda-\omega}{\omega} Z_{m a-s / 2}(\lambda z) w^{(\sigma)}
\end{array}\right),
\end{aligned}
$$

where $\mathbf{k}=\left(k_{1}, \ldots, k_{D-1}\right), \omega=\sqrt{\lambda^{2}+k^{2}}, k=|\mathbf{k}|, \mathbf{k x}=$ $\sum_{l=1}^{D-1} k_{i} x^{i}$, and $\mathbf{k} \chi=\sum_{l=1}^{D-1} k_{l} \chi_{l}$. In (3.3), $Z_{\mu}(\lambda z)$, with $\mu=m a \pm s / 2$, is a cylinder function. We present it in the form of a linear combination of the Bessel and Neumann functions $J_{\mu}(\lambda z)$ and $Y_{\mu}(\lambda z)$ :

$$
Z_{\mu}(\lambda z)=c_{1} J_{\mu}(\lambda z)+c_{2} Y_{\mu}(\lambda z),
$$

where the coefficients are determined by the normalization and boundary conditions.

The momentum $\mathbf{k}$ in the mode functions (3.3) can be decomposed as $\mathbf{k}=\left(\mathbf{k}_{(p)}, \mathbf{k}_{(q)}\right)$, where $\mathbf{k}_{(p)}=\left(k_{1}, \ldots, k_{p}\right)$ and $\mathbf{k}_{(q)}=\left(k_{p+1}, \ldots, k_{D-1}\right)$ correspond to the uncompact and compact subspaces. For the components in the uncompact subspace, as usual, one has $-\infty<k_{i}<+\infty$, $i=1, \ldots, p$, whereas the components in the compact subspace are discretized by the periodicity conditions (2.3) (with the replacement $\alpha_{l} \rightarrow \tilde{\alpha}_{l}$ in the gauge under consideration) with the eigenvalues

$$
k_{l}=\frac{2 \pi n_{l}+\tilde{\alpha}_{l}}{L_{l}}, \quad n_{l}=0, \pm 1, \pm 2, \ldots,
$$

and $l=p+1, \ldots, D-1$. In (3.5), the integer part of the ratio $\tilde{\alpha}_{l} / 2 \pi$ can be absorbed by the redefinition of $n_{l}$ and only the fractional part of that ratio is physically relevant.

Now let us consider the boundary conditions on the branes. From (2.5) it follows that $Z_{m a+1 / 2}\left(\lambda z_{1}\right)=0$ and
$Z_{m a-1 / 2}\left(\lambda z_{2}\right)=0$ in both the cases $s= \pm 1$. For the ratio of the coefficients in (3.4) the first condition gives

$$
\frac{c_{2}}{c_{1}}=-\frac{J_{m a+1 / 2}\left(\lambda z_{1}\right)}{Y_{m a+1 / 2}\left(\lambda z_{1}\right)}
$$

From the second condition we obtain that the allowed values of $\lambda$ are roots of the equation

$$
g_{m a+1 / 2, m a-1 / 2}\left(\lambda z_{1}, \lambda z_{2}\right)=0,
$$

where we have introduced the function

$$
g_{\mu, \nu}(x, u)=J_{\mu}(x) Y_{\nu}(u)-J_{\nu}(u) Y_{\mu}(x) .
$$

It can be seen that the Eq. (3.7) has no solutions corresponding to bound states with $\lambda=i \xi, \xi>0$. ${ }^{1}$ We denote the positive roots of (3.7) with respect to $\lambda z_{1}$ by $\lambda_{n}=\lambda_{n}\left(m a, z_{2} / z_{1}\right)=\lambda z_{1}, n=1,2, \ldots$, assuming that they are numerated in the ascending order, $\lambda_{n+1}>\lambda_{n}$. Note that the roots $\lambda_{n}$ depend on the locations of the branes in the form of the ratio $z_{2} / z_{1}$. For a massless field the Eq. (3.7) is reduced to $\cos \left[\lambda\left(z_{2}-z_{1}\right)\right]=0$ with the solutions $\lambda z_{1}=$ $\lambda_{n}=\pi(n-1 / 2) /\left(z_{2} / z_{1}-1\right), n=1, \ldots, \infty$. These eigenvalues coincide with those for parallel plates in the locally Minkowski bulk located at $z=z_{1}$ and $z=z_{2}$. For large values of $\lambda z_{1}$ and for a massive field, in (3.7) we can use the asymptotic expressions for the cylinder functions for large arguments. To the leading order the lefthand side of (3.7) is reduced to $\cos \left[\lambda\left(z_{2}-z_{1}\right)\right]$. Hence, for large $n$ one has $\lambda_{n} \approx \pi(n-1 / 2) /\left(z_{2} / z_{1}-1\right)$. The mode functions are specified by the set $\beta=\left(\mathbf{k}_{(p)}, \mathbf{n}_{q}, n, \sigma\right)$ with $\mathbf{n}_{q}=\left(n_{p+1}, \ldots, n_{D-1}\right)$.

The function $Z_{\nu}(\lambda z)$ is expressed in terms of the function (3.8) in two equivalent ways:

$Z_{\mu}(\lambda z)=-\frac{c_{1} g_{m a+1 / 2, \mu}\left(\lambda z_{1}, \lambda z\right)}{Y_{m a+1 / 2}\left(\lambda z_{1}\right)}=-\frac{c_{1} g_{m a-1 / 2, \mu}\left(\lambda z_{2}, \lambda z\right)}{Y_{m a-1 / 2}\left(\lambda z_{2}\right)}$.

By using the first of these relations, and introducing the notation

$$
\nu=m a+1 / 2
$$

for the further convenience, the mode functions are presented in the form

\footnotetext{
${ }^{1}$ In the geometry of a single brane at $z=z_{j}$ and in the region $z_{j}<z<\infty$ the corresponding normalizable mode functions would be given by (3.3) with the functions $Z_{m a \pm s / 2}(\lambda z)$ replaced by the Macdonald functions $K_{m a \pm s / 2}(\xi z)$ (up to the normalization constant). They exponentially decay at large distances from the brane and correspond to bound states.
} 


$$
\begin{aligned}
\psi_{\beta}^{(+)}(x)= & C_{\beta}^{(+)} z^{\frac{D+1}{2}} e^{i \mathbf{k x}-i \omega t} \\
& \times\left(\begin{array}{c}
\frac{\mathbf{k} \chi \chi_{0}^{\dagger}+i \lambda-\omega}{\omega} g_{\nu, m a+s / 2}\left(\lambda z_{1}, \lambda z\right) w^{(\sigma)} \\
i \chi_{0}^{\dagger} \frac{\mathbf{k} \chi_{0}^{\dagger}+i \lambda+\omega}{\omega} g_{\nu, m a-s / 2}\left(\lambda z_{1}, \lambda z\right) w^{(\sigma)}
\end{array}\right), \\
\psi_{\beta}^{(-)}(x)= & C_{\beta}^{(-)} z^{\frac{D+1}{2}} e^{i \mathbf{k} \mathbf{x}+i \omega t} \\
& \times\left(\begin{array}{c}
i \chi_{0} \frac{\mathbf{k} \chi^{\dagger} \chi_{0}-i \lambda+\omega}{\omega} g_{\nu, m a+s / 2}\left(\lambda z_{1}, \lambda z\right) w^{(\sigma)} \\
\frac{\mathbf{k} \chi^{\dagger} \chi_{0}-i \lambda-\omega}{\omega} g_{\nu, m a-s / 2}\left(\lambda z_{1}, \lambda z\right) w^{(\sigma)}
\end{array}\right),
\end{aligned}
$$

with $\lambda=\lambda_{n} / z_{1}$ and new normalization coefficients $C_{\beta}^{( \pm)}$. The latter are found from the normalization condition

$$
\int d^{D} x(a / z)^{D} \psi_{\beta}^{( \pm) \dagger} \psi_{\beta^{\prime}}^{( \pm)}=\delta_{\beta \beta^{\prime}}
$$

where the $z$-integration goes over the range $\left[z_{1}, z_{2}\right], \delta_{\beta \beta^{\prime}}$ is understood as Kronecker delta for the discrete components of the collective index $\beta$ and as Dirac delta function for the continuous ones. The normalization integral over $z$ contains the squares of the functions $g_{\nu, m a \pm 1 / 2}\left(\lambda z_{1}, \lambda z\right)$. By taking into account that these functions are cylinder functions with respect to both the arguments, the integrals are evaluated by using the corresponding formula from [30]. Using the fact that $\lambda$ is a root of the Eq. (3.7), it can be shown that

$$
\int_{z_{1}}^{z_{2}} d z z g_{\nu, m a \pm 1 / 2}^{2}\left(\lambda z_{1}, \lambda z\right)=\frac{2 \pi^{-2} z_{1} / \lambda}{T_{\nu}\left(z_{2} / z_{1}, \lambda z_{1}\right)},
$$

where we have defined

$$
T_{\nu}(\eta, x)=x\left[\frac{J_{\nu}^{2}(x)}{J_{\nu-1}^{2}(\eta x)}-1\right]^{-1}, \quad \eta=z_{2} / z_{1} .
$$

On the base (3.13) it can be seen that

$$
\left|C_{\beta}^{( \pm)}\right|^{2} \equiv\left|C_{\beta}\right|^{2}=\frac{\lambda T_{\nu}\left(\eta, \lambda z_{1}\right)}{32(2 \pi)^{p-2} V_{q} a^{D} z_{1}} .
$$

As seen the normalization constants are the same (up to a phase) for the positive and negative energy solutions and they do not depend on the parameter $s$.

In the absence of branes, in AdS spacetime two classes of field modes are present: normalizable and non-normalizable. The non-normalizability of the second class of modes comes from their diverging behavior on the AdS boundary at $z=0$. In the field quantization procedure the normalizable modes are used to built up the Hilbert space. The non-normalizable modes are not part of the Hilbert space and they are considered as defining background. In AdS/CFT correspondence the non-normalizable modes correspond to classical sources in the theory on the AdS boundary. In the problem under consideration the field is causally separated from the AdS boundary by the brane at $z=z_{1}>0$. As a consequence of that all the modes of the fermionic field in the region $z_{1} \leq z \leq z_{2}$ are regular and there are no non-normalizable modes. A similar situation is realized in Randall-Sundrum braneworld models with two branes where a slice of the AdS spacetime is employed.

\section{CURRENT DENSITY IN THE REGION BETWEEN THE BRANES}

Having the complete set of normalized mode functions (3.11), we can evaluate the VEV of the current density $j^{\mu}=e \bar{\psi} \gamma^{\mu} \psi$, where $\bar{\psi}=\psi^{\dagger} \gamma^{(0)}$ is the Dirac conjugate (for a recent discussion of the renormalized fermion expectation values on $\mathrm{AdS}$ spacetime in the absence of branes see, for example, [31]). That is done by using the mode-sum formula

$$
\left\langle j^{\mu}(x)\right\rangle=\frac{e}{2} \sum_{\beta}\left[\bar{\psi}_{\beta}^{(-)}(x) \gamma^{\mu} \psi_{\beta}^{(-)}(x)-\bar{\psi}_{\beta}^{(+)}(x) \gamma^{\mu} \psi_{\beta}^{(+)}(x)\right],
$$

where $\left\langle j^{\mu}(x)\right\rangle=\left\langle 0\left|j^{\mu}(x)\right| 0\right\rangle$ with $|0\rangle$ being the vacuum state, and

$$
\sum_{\beta}=\sum_{\mathbf{n}_{q}} \int d \mathbf{k}_{(p)} \sum_{n=1}^{\infty} \sum_{\sigma=1}^{N / 2} .
$$

We consider the charge density and the spatial components separately.

The component with $\mu=0$ in (4.1) corresponds to the VEV of the charge density. Inserting the mode functions it is presented in the form

$$
\begin{aligned}
\left\langle j^{0}\right\rangle= & \frac{(2 \pi)^{2-p} e z^{D+2}}{16 a^{D+1} V_{q} z_{1}} \sum_{\beta} \frac{\lambda}{\omega} T_{\nu}\left(\eta, \lambda z_{1}\right) \\
& \times \sum_{j= \pm 1} j g_{\nu, m a+j s / 2}^{2}\left(\lambda z_{1}, \lambda z\right) w^{(\sigma) \dagger} \mathbf{k} \chi^{\dagger} \chi_{0} w^{(\sigma)} .
\end{aligned}
$$

From the definition of the one-column matrices $w^{(\sigma)}$ it follows that $\sum_{\sigma=1}^{N / 2} w^{(\sigma) \dagger} \chi^{\dagger} \chi_{0} w^{(\sigma)}=\operatorname{tr}\left(\chi^{\dagger} \chi_{0}\right)$. Now, by taking into account the commutation relations for the matrices $\chi_{0}$ and $\chi_{b}$, we can show that $\operatorname{tr}\left(\chi_{b}^{\dagger} \chi_{0}\right)=0$ and consequently $\sum_{\sigma=1}^{N / 2} w^{(\sigma) \dagger} \chi^{\dagger} \chi_{0} w^{(\sigma)}=0$. Hence, we conclude that the VEV of the charge density vanishes.

Next we consider the spatial components of the VEV (4.1). With the mode functions (3.11) we get

$$
\begin{aligned}
\left\langle j^{l}\right\rangle= & -\frac{(2 \pi)^{2-p} e N z^{D+2}}{32 V_{q} a^{D+1} z_{1}} \sum_{\mathbf{n}_{q}} \int d \mathbf{k}_{(p)} k_{l} \sum_{n=1}^{\infty} \frac{\lambda}{\omega} T_{\nu}\left(\eta, \lambda z_{1}\right) \\
& \times \sum_{j= \pm 1} g_{\nu, m a+j s / 2}^{2}\left(\lambda z_{1}, \lambda z\right) .
\end{aligned}
$$


For the components along uncompact dimensions, $l=1, \ldots, p$, in (4.4) one has $-\infty<k_{l}<+\infty$ and in the integral $\int_{-\infty}^{+\infty} d k_{l}$ the integrand is an odd function of the integration variable. From here we conclude that the components of the current density along uncompact dimensions vanish: $\left\langle j^{l}\right\rangle=0$ for $l=1, \ldots, p$. This result could also be directly obtained on the base of the problem symmetry under the reflections $x^{l} \rightarrow-x^{l}$ of the uncompact directions. Hence, a nonzero vacuum currents may appear along the compact dimensions only and we pass to the investigation of their properties.

\section{A. Integral representation for the currents in the compact subspace}

First of all, from (4.4) it follows that the current density does not depend on the parameter $s$. This means that in odddimensional spacetimes the current densities are the same for fermionic fields realizing two inequivalent irreducible representations of the Clifford algebra. In the following discussion we put $s=1$. After integrating over the angular part of $\mathbf{k}_{(p)}$, the current density along the $l$ th compact dimension is presented in the form

$$
\begin{aligned}
\left\langle j^{l}\right\rangle= & -\frac{\pi^{2-p / 2} e N z^{D+2}}{2^{p+2} \Gamma(p / 2) V_{q} a^{D+1} z_{1}} \sum_{\mathbf{n}_{q}} k_{l} \int_{0}^{\infty} d k_{(p)} k_{(p)}^{p-1} \\
& \times \sum_{n=1}^{\infty} \frac{\lambda_{n} T_{\nu}\left(\eta, \lambda_{n}\right)}{\sqrt{\lambda_{n}^{2}+z_{1}^{2} k^{2}}} \sum_{j= \pm 1} g_{\nu, m a+j / 2}^{2}\left(\lambda_{n}, \lambda_{n} z / z_{1}\right),
\end{aligned}
$$

where $l=p+1, \ldots, D-1, k^{2}=k_{(p)}^{2}+k_{(q)}^{2}, k_{(p)}^{2}=\left|\mathbf{k}_{(p)}\right|^{2}$ and

$$
k_{(q)}^{2}=\sum_{i=p+1}^{D-1} \frac{\left(2 \pi n_{i}+\tilde{\alpha}_{i}\right)^{2}}{L_{i}^{2}} .
$$

From (4.5) it follows that $\left\langle j^{l}\right\rangle$ is an odd periodic function of $\tilde{\alpha}_{l}$ with the period $2 \pi$ and an even periodic function of $\tilde{\alpha}_{i}$, $i \neq l$, with the same period. In terms of the magnetic fluxes $\Phi_{i}$ this means that the current density is a periodic function of the magnetic fluxes with the period equal to the flux quantum. In particular, $\left\langle j^{l}\right\rangle$ vanishes for integer values of $\tilde{\alpha}_{l} /(2 \pi)$. The charge flux density through the hypersurface $x^{l}=$ const is given by $n_{l}^{(l)}\left\langle j^{l}\right\rangle$, where $n_{i}^{(l)}=\delta_{i}^{l} a / z$ is the normal to that hypersurface. The product $a^{D} n_{l}^{(l)}\left\langle j^{l}\right\rangle$ depends on the variables having the dimension of length in the form of the dimensionless combinations $z_{j} / z, L_{i} / z, m a$. This feature is a consequence of the maximal symmetry of the AdS spacetime. In figures below we plot the quantity $a^{D} n_{l}^{(l)}\left\langle j^{l}\right\rangle$.

In the representation (4.5) the eigenvalues $\lambda_{n}$ are given implicitly, as roots of the Eq. (3.7). Another disadvantage is that the terms with large $n$ are highly oscillatory. A more convenient representation is obtained applying a variant of the generalized Abel-Plana formula (A1), derived in Appendix, with $\mu=\nu-1$ and $\delta=1$. Note that $\lambda_{\nu-1, n}^{(1)}=\lambda_{n}$ and $T_{\nu-1}^{(1)}(\eta, x)=T_{\nu}(\eta, x)$. For the series over $n$ in (4.5) the function $h(u)$ has the form

$$
h(x)=\frac{x}{\sqrt{x^{2}+z_{1}^{2} k^{2}}} \sum_{j= \pm 1} g_{\nu, m a+j / 2}^{2}\left(x, x z / z_{1}\right),
$$

and has branch points $x= \pm i z_{1} k$ on the imaginary axis. By using the properties of the Bessel functions it can be seen that $h(i x)+h(-i x)=0$ for $0 \leq x<z_{1} k$. With the help of (A1) the current density is presented as

$$
\begin{aligned}
\left\langle j^{l}\right\rangle & =\left\langle j^{l}\right\rangle^{(1)}-\frac{4(4 \pi)^{-p / 2-1} e N z^{D+2}}{\Gamma(p / 2) V_{q} a^{D+1} z_{1}} \sum_{\mathbf{n}_{q}} k_{l} \int_{0}^{\infty} d k_{(p)} k_{(p)}^{p-1} \\
& \times \int_{z_{1} k}^{\infty} d x x \frac{K_{\nu-1}(\eta x)}{K_{\nu}(x)} \frac{\sum_{j= \pm 1} j G_{\nu, m a+j / 2}^{2}\left(x, x z / z_{1}\right)}{\sqrt{x^{2}-z_{1}^{2} k^{2}} G_{\nu, \nu-1}(x, \eta x)},
\end{aligned}
$$

where we have defined

$$
G_{\mu, \nu}(x, u)=I_{\mu}(x) K_{\nu}(u)-(-1)^{\mu-\nu} K_{\mu}(x) I_{\nu}(u) .
$$

with the modified Bessel functions $I_{\mu}(x)$ and $K_{\mu}(x)$. The first term in the right-hand side of (4.8) is given by

$$
\begin{aligned}
\left\langle j^{l}\right\rangle^{(1)}= & -\frac{(4 \pi)^{-p / 2} e N z^{D+2}}{2 \Gamma(p / 2) V_{q} a^{D+1} z_{1}} \sum_{\mathbf{n}_{q}} k_{l} \int_{0}^{\infty} d k_{(p)} k_{(p)}^{p-1} \\
& \times \int_{0}^{\infty} d x \frac{x}{\sqrt{x^{2}+z_{1}^{2} k^{2}}} \frac{\sum_{j= \pm 1} g_{\nu, m a+j / 2}^{2}\left(x, x z / z_{1}\right)}{J_{\nu}^{2}(x)+Y_{\nu}^{2}(x)},
\end{aligned}
$$

and it corresponds to the current density in the region $z_{1} \leq$ $z<\infty$ for the geometry of a single brane located at $z=z_{1}$.

The single brane part (4.10) has been investigated in [29]. It is decomposed as

$$
\left\langle j^{l}\right\rangle^{(1)}=\left\langle j^{l}\right\rangle_{0}+\left\langle j^{l}\right\rangle_{b}^{(1)},
$$

where the term $\left\langle j^{l}\right\rangle_{0}$ is the current density in the absence of the branes and

$$
\begin{aligned}
\left\langle j^{l}\right\rangle_{b}^{(1)}= & \frac{N e A_{p} z^{D+2}}{V_{q} a^{D+1}} \sum_{\mathbf{n}_{q}} k_{l} \int_{k_{(q)}}^{\infty} d u u\left(u^{2}-k_{(q)}^{2}\right)^{\frac{p-1}{2}} \\
& \times \frac{I_{\nu}\left(z_{1} u\right)}{K_{\nu}\left(z_{1} u\right)} \sum_{j= \pm 1} j K_{m a+j / 2}^{2}(z u)
\end{aligned}
$$

with 


$$
A_{p}=\frac{(4 \pi)^{-(p+1) / 2}}{\Gamma((p+1) / 2)}
$$

is the part induced in the region $z \geq z_{1}$ by a single brane at $z=z_{1}$. The part $\left\langle j^{l}\right\rangle_{0}$ is investigated in [25]. It is expressed in terms of the function

$$
q_{\mu}^{(D+1) / 2}(u)=\sqrt{\frac{\pi}{2}} \int_{0}^{\infty} d x x^{D / 2} e^{-u x} I_{\mu+1 / 2}(x),
$$

and is presented in the form

$$
\begin{aligned}
\left\langle j^{l}\right\rangle_{0}= & -\frac{e N a^{-D-1} L_{l}}{(2 \pi)^{(D+1) / 2}} \sum_{n_{l}=1}^{\infty} n_{l} \sin \left(\tilde{\alpha}_{l} n_{l}\right) \sum_{\mathbf{n}_{q-1}} \cos \left(\sum_{i=1, \neq l}^{D-1} \tilde{\alpha}_{i} n_{i}\right) \\
& \times \sum_{j=0,1} q_{m a-j}^{(D+1) / 2}\left(1+\sum_{i=p+1}^{D-1} \frac{n_{i}^{2} L_{i}^{2}}{2 z^{2}}\right),
\end{aligned}
$$

where $\mathbf{n}_{q-1}=\left(n_{p+1}, \ldots, n_{l-1}, n_{l+1}, \ldots n_{D-1}\right)$. An alternative expression for the function (4.14) in terms of the hypergeometric function is given in [25].

The last term in (4.8) is induced in the region $z_{1} \leq z \leq z_{2}$ if we add to the geometry of a single brane at $z=z_{1}$ the second brane at $z=z_{2}$. It can be further transformed by introducing a new integration variable $w=\sqrt{x^{2}-z_{1}^{2} k^{2}}$ and passing to polar coordinates $(r, \theta)$ in the plane $\left(z_{1} k_{(p)}, w\right)$. After integrating over $\theta$ and introducing instead of $r$ the integration variable $u=\sqrt{r^{2} / z_{1}^{2}+k_{(q)}^{2}}$, we find

$$
\begin{aligned}
\left\langle j^{l}\right\rangle= & \left\langle j^{l}\right\rangle_{0}+\left\langle j^{l}\right\rangle_{b}^{(1)}-\frac{N e A_{p} z^{D+2}}{V_{q} a^{D+1}} \sum_{\mathbf{n}_{q}} k_{l} \int_{k_{(q)}}^{\infty} d u u \frac{K_{\nu-1}\left(z_{2} u\right)}{K_{\nu}\left(z_{1} u\right)} \\
& \times \frac{\left(u^{2}-k_{(q)}^{2}\right)^{\frac{p-1}{2}}}{G_{\nu, \nu-1}\left(z_{1} u, z_{2} u\right)} \sum_{j= \pm 1} j G_{\nu, m a+j / 2}^{2}\left(z_{1} u, z u\right)
\end{aligned}
$$

By taking into account the representation (4.11) for a single brane part, the current density is presented as

$$
\begin{aligned}
\left\langle j^{l}\right\rangle= & \left\langle j^{l}\right\rangle_{0}+\frac{N e A_{p} z^{D+2}}{V_{q} a^{D+1}} \sum_{\mathbf{n}_{q}} k_{l} \int_{k_{(q)}}^{\infty} d u u\left(u^{2}-k_{(q)}^{2}\right)^{\frac{p-1}{2}} \\
& \times\left[\frac{K_{\nu}\left(z_{1} u\right) I_{\nu-1}\left(z_{2} u\right)}{I_{\nu}\left(z_{1} u\right) K_{\nu-1}\left(z_{2} u\right)}+1\right]^{-1} \\
& \times \sum_{j= \pm 1} j\left[\frac{I_{\nu-1}\left(z_{2} u\right)}{K_{\nu-1}\left(z_{2} u\right)} K_{m a+j / 2}^{2}(z u)-\frac{K_{\nu}\left(z_{1} u\right)}{I_{\nu}\left(z_{1} u\right)} I_{m a+j / 2}^{2}(z u)\right. \\
& \left.+2 j K_{m a+j / 2}(z u) I_{m a+j / 2}(z u)\right] .
\end{aligned}
$$

An alternative representation is given by the formula

$$
\begin{aligned}
\left\langle j^{l}\right\rangle= & \left\langle j^{l}\right\rangle_{0}+\left\langle j^{l}\right\rangle_{b}^{(2)}+\frac{N e A_{p} z^{D+2}}{V_{q} a^{D+1}} \sum_{\mathbf{n}_{q}} k_{l} \int_{k_{(q)}}^{\infty} d u u \frac{I_{\nu}\left(z_{1} u\right)}{I_{\nu-1}\left(z_{2} u\right)} \\
& \times \frac{\left(u^{2}-k_{(q)}^{2}\right)^{\frac{p-1}{2}}}{G_{\nu, \nu-1}\left(z_{1} u, z_{2} u\right)} \sum_{j= \pm 1} j G_{\nu-1, m a+j / 2}^{2}\left(z_{2} u, z u\right),
\end{aligned}
$$

where

$$
\begin{aligned}
\left\langle j^{l}\right\rangle_{b}^{(2)}= & -\frac{N e A_{p} z^{D+2}}{V_{q} a^{D+1}} \sum_{\mathbf{n}_{q}} k_{l} \int_{k_{(q)}}^{\infty} d u u\left(u^{2}-k_{(q)}^{2}\right)^{\frac{p-1}{2}} \\
& \times \frac{K_{\nu-1}\left(u z_{2}\right)}{I_{\nu-1}\left(u z_{2}\right)} \sum_{j= \pm 1} j I_{m a+j / 2}^{2}(u z),
\end{aligned}
$$

is the current density induced by a single brane at $z=z_{2}$ in the region $0 \leq z \leq z_{2}$ (see [29]). The last term in (4.18) is induced by the brane at $z=z_{1}$ if we add it to the problem with a single brane at $z=z_{2}$. As it will be seen in the next subsection, the VEV of the current density is finite on the branes. However, in the representations (4.12) and (4.19) for single brane-induced parts we cannot directly put in the integrands $z=z_{1}$ and $z=z_{2}$, respectively. This can be done in the second brane-induced contributions [last terms in (4.16) and (4.18)].

In order to see the interference effects between the branes we can present the total current density as

$$
\left\langle j^{l}\right\rangle=\left\langle j^{l}\right\rangle_{0}+\left\langle j^{l}\right\rangle_{b}^{(1)}+\left\langle j^{l}\right\rangle_{b}^{(2)}+\left\langle j^{l}\right\rangle_{\text {int }} .
$$

By taking into account the expressions for the single brane parts, for the interference part we can get the expression

$$
\begin{aligned}
\left\langle j^{l}\right\rangle_{\mathrm{int}}= & \frac{N e A_{p} z^{D+2}}{V_{q} a^{D+1}} \sum_{\mathbf{n}_{q}} k_{l} \int_{k_{(q)}}^{\infty} d u u\left(u^{2}-k_{(q)}^{2}\right)^{\frac{p-1}{2}} \\
& \times\left[\frac{K_{\nu}\left(z_{1} u\right) I_{\nu-1}\left(z_{2} u\right)}{I_{\nu}\left(z_{1} u\right) K_{\nu-1}\left(z_{2} u\right)}+1\right]^{-1} \\
& \times \sum_{j= \pm 1} j\left[\frac{K_{\nu-1}\left(z_{2} u\right)}{I_{\nu-1}\left(z_{2} u\right)} I_{m a+j / 2}^{2}(z u)\right. \\
& -\frac{I_{\nu}\left(z_{1} u\right)}{K_{\nu}\left(z_{1} u\right)} K_{m a+j / 2}^{2}(z u) \\
& \left.+2 j K_{m a+j / 2}(z u) I_{m a+j / 2}(z u)\right]
\end{aligned}
$$

Note that in the evaluation of the interference part on the branes we can directly put in the integrand $z=z_{j}$.

The current densities in the regions $z \leq z_{1}$ and $z \geq z_{2}$ coincide with those in the corresponding geometries with single branes. In these regions the VEV is presented as $\left\langle j^{l}\right\rangle=\left\langle j^{l}\right\rangle_{0}+\left\langle j^{l}\right\rangle_{b}$, where the brane-induced contribution $\left\langle j^{l}\right\rangle_{b}$ is given by (4.19) in the region $z \leq z_{1}$, with the 
replacement $z_{2} \rightarrow z_{1}$, and by (4.12) in the region $z \geq z_{2}$, with the replacement $z_{1} \rightarrow z_{2}$.

\section{B. Alternative representation and the currents on the branes}

Here we provide another representation for the VEV of the current density that is more adapted for the investigation of the near-brane asymptotics. It is obtained from the initial expression (4.5) by using the summation formula [16]

$$
\begin{aligned}
\frac{2 \pi}{L_{l}} \sum_{n_{l}=-\infty}^{\infty} g\left(k_{l}\right) f\left(\left|k_{l}\right|\right)= & \int_{0}^{\infty} d u[g(u)+g(-u)] f(u) \\
& +i \int_{0}^{\infty} d u[f(i u)-f(-i u)] \\
& \times \sum_{j= \pm 1} \frac{g(i j u)}{e^{u L_{l}+i j \tilde{\alpha}_{l}}-1},
\end{aligned}
$$

where $k_{l}$ is given by (3.5). In the special case $g(x)=1$, $\tilde{\alpha}_{l}=0$ the standard Abel-Plana formula is obtained from (4.22). For the series over $n_{l}$ in (4.5) we have $g(u)=u$ and the first integral in the right-hand side of (4.22) vanishes. Physically this corresponds to the fact that the part in the current density with that integral presents the current in the model where the dimension $x^{l}$ is decompactified and, hence, as it has been shown above, the corresponding current is zero. With $g(u)=u$, by using the expansion $1 /\left(e^{y}-1\right)=\sum_{r=1}^{\infty} e^{-r y}$, after evaluating the integrals over $u$ and $k_{(p)}$, one gets

$$
\begin{aligned}
\left\langle j^{l}\right\rangle= & -\frac{(2 \pi)^{1-p / 2} e N z^{D+2}}{8 V_{q} L_{l}^{p} a^{D+1} z_{1}^{2}} \sum_{r=1}^{\infty} \frac{\sin \left(r \tilde{\alpha}_{l}\right)}{r^{p+1}} \sum_{\mathbf{n}_{q-1}} \sum_{n=1}^{\infty} \lambda_{n} T_{\nu}\left(\eta, \lambda_{n}\right) \\
& \times g_{p / 2+1}\left(r L_{l} \sqrt{\lambda_{n}^{2} / z_{1}^{2}+k_{(q-1)}^{2}}\right) \sum_{j= \pm 1} g_{\nu, m a+j / 2}^{2}\left(\lambda_{n}, \lambda_{n} z / z_{1}\right) .
\end{aligned}
$$

where $k_{(q-1)}^{2}=\sum_{i=p+1, \neq l}^{D-1}\left(2 \pi n_{i}+\tilde{\alpha}_{i}\right)^{2} / L_{i}^{2}$ and we have defined the function

$$
g_{\mu}(x)=x^{\mu} K_{\mu}(x) .
$$

Unlike to the series over $n$ in (4.5) the corresponding series in (4.23) is exponentially convergent.

The representation (4.23) is well adapted for the investigation of the currents on the branes. They are obtained putting $z=z_{j}$ directly in the right-hand side of (4.23). By taking into account that $g_{\nu, \nu-1}\left(\lambda_{n}, \eta \lambda_{n}\right)=0$, and

$$
\begin{aligned}
g_{\nu, \nu-1}\left(\lambda_{n}, \lambda_{n}\right) & =\frac{2}{\pi \lambda_{n}}, \\
g_{\nu, \nu}\left(\lambda_{n}, \eta \lambda_{n}\right) & =-\frac{2}{\pi \eta \lambda_{n}} \frac{J_{\nu}\left(\lambda_{n}\right)}{J_{\nu-1}\left(\eta \lambda_{n}\right)},
\end{aligned}
$$

one gets

$$
\begin{aligned}
\left\langle j^{l}\right\rangle_{z=z_{j}}= & -\frac{2 e N L_{l}^{-p} z_{j}^{D}}{(2 \pi)^{p / 2+1} V_{q} a^{D+1}} \sum_{r=1}^{\infty} \frac{\sin \left(r \tilde{\alpha}_{l}\right)}{r^{p+1}} \\
& \times \sum_{n=1}^{\infty}\left[\frac{J_{\nu}\left(\lambda_{n}\right)}{J_{\nu-1}\left(\eta \lambda_{n}\right)}\right]^{2(j-1)} \\
& \times \sum_{\mathbf{n}_{q-1}} \frac{g_{p / 2+1}\left(r L_{l} \sqrt{\left.\lambda_{n}^{2} / z_{1}^{2}+k_{(q-1)}^{2}\right)}\right.}{J_{\nu}^{2}\left(\lambda_{n}\right) / J_{\nu-1}^{2}\left(\eta \lambda_{n}\right)-1} .
\end{aligned}
$$

Finiteness of the vacuum current density on the branes is in clear contrast with the corresponding behavior of the fermionic condensate and of the VEV of the energymomentum tensor. The latter VEVs diverge on the boundaries. This kind of surface divergences have been widely discussed in the Casimir effect for fields with different spins and for different boundary geometries. The absence of the surface divergences for the current density in the problem under consideration can be understood from general arguments. In the problem with two branes and without compact dimensions the VEV of the current density vanishes. The compactification scheme we have considered does not change the local bulk and boundary geometries. By taking into account that the divergences are completely determined by those local geometries, we conclude that the toral compactification will not induce additional divergences in the VEVs. In particular, the VEV of the current density becomes finite everywhere.

We can also use the representation (4.23) for the evaluation of the total current, per unit surface along the uncompact dimensions. By using the integrals (3.13) we get

$$
\begin{aligned}
V_{q} \int_{z_{1}}^{z_{2}} d z \sqrt{|g|}\left\langle j^{l}\right\rangle= & -\frac{2 N e}{(2 \pi)^{p / 2+1} L_{l}^{p}} \sum_{r=1}^{\infty} \frac{\sin \left(r \tilde{\alpha}_{l}\right)}{r^{p+1}} \\
& \times \sum_{\mathbf{n}_{q-1}} \sum_{n=1}^{\infty} g_{p / 2+1}\left(r L_{l} \sqrt{\lambda_{n}^{2} / z_{1}^{2}+k_{(q-1)}^{2}}\right),
\end{aligned}
$$

where $g$ is the determinant of the metric tensor. In (4.27), the information on the curvature and on the boundary geometry is encoded through the ratio $\lambda_{n} / z_{1}$. For a given distance between the branes the ratio $z_{2} / z_{1}$ is fixed and the roots $\lambda_{n}$ do not depend on the location of the left brane $z_{1}$. In particular, from here it follows that, for fixed $z_{2} / z_{1}$, the quantity (4.27) goes to zero in the limit $z_{1} \rightarrow 0$. Comparing the integrated current (4.27) with the current densities (4.26) on the branes, the following simple relation between them is obtained. 


$$
\int_{z_{1}}^{z_{2}} d z \sqrt{|g|}\left\langle j^{l}\right\rangle=\left[\frac{a^{D+1}}{z^{D}}\left\langle j^{l}\right\rangle\right]_{z=z_{1}}^{z=z_{2}} .
$$

In the model with a single compact dimension $x^{l}$ with the length $L_{l}(q=1, p=D-2, l=D-1)$ the formula (4.23) is specified to

$$
\begin{aligned}
\left\langle j^{l}\right\rangle= & -\frac{(2 \pi)^{-D / 2} e N z^{D+2}}{8 L_{l}^{D-1} a^{D+1} z_{1}^{2}} \sum_{r=1}^{\infty} \frac{\sin \left(r \tilde{\alpha}_{l}\right)}{r^{D-1}} \sum_{n=1}^{\infty} \lambda_{n} T_{\nu}\left(\eta, \lambda_{n}\right) \\
& \times g_{D / 2}\left(r L_{l} \lambda_{n} / z_{1}\right) \sum_{j= \pm 1} g_{\nu, m a+j / 2}^{2}\left(\lambda_{n}, \lambda_{n} z / z_{1}\right) .
\end{aligned}
$$

An alternative expression in this special case is obtained from (4.17). In this and in the next sections, for numerical investigations of the current density we consider the special case $D=4$ with a single compact dimension of the length $L_{l}=L$ and with the phase in the periodicity condition $\tilde{\alpha}_{l}=\tilde{\alpha}$. For this model the corresponding formulas are obtained from (4.17) and (4.29) taking $p=2$ and $q=1$.

\section{Asymptotics and numerical examples}

In this subsection we consider the behavior of the current density in asymptotic regions of the parameters. The Minkowskian limit corresponds to $a \rightarrow \infty$ for fixed $y$ and $y_{j}$. In this limit the conformal coordinates $z$ and $z_{j}$ are large, $z \approx a+y, z-z_{j} \approx y-y_{j}$, and, consequently, both the order and the argument of the modified Bessel functions in (4.17) are large. By using the corresponding uniform asymptotic expansions [32], for the brane-induced part, to the leading order, we get $\left\langle j^{l}\right\rangle-\left\langle j^{l}\right\rangle_{0} \approx\left\langle j^{l}\right\rangle_{b}^{(M)}$, where

$$
\begin{aligned}
\left\langle j^{l}\right\rangle_{b}^{(M)}= & \frac{2 N e A_{p}}{V_{q}} \sum_{\mathbf{n}_{q}} k_{l} \int_{m_{(q)}}^{\infty} d x \frac{\left(x^{2}-m_{(q)}^{2}\right)^{\frac{p-1}{2}}}{\frac{x+m}{x-m} e^{2 x\left(y_{2}-y_{1}\right)}+1} \\
& \times\left\{1+\frac{m e^{x\left(y_{2}-y_{1}\right)}}{x-m} \cosh \left[x\left(2 y-y_{2}-y_{1}\right)\right]\right\},
\end{aligned}
$$

with the notation $m_{(q)}=\sqrt{m^{2}+k_{(q)}^{2}}$. This expression coincides with the result from [18] for two boundaries in a flat bulk with topology $R^{p+1} \times T^{q}$ (with the sign difference related to definition of the parameters $\tilde{\alpha}_{i}$ ).

For a massless fermionic field the modified Bessel functions in (4.16) are expressed in terms of the elementary functions. By taking into account the expressions

$$
\begin{aligned}
G_{1 / 2,1 / 2}(x, y) & =\frac{\sinh (x-y)}{\sqrt{x y}}, \\
G_{1 / 2,-1 / 2}(x, y) & =\frac{\cosh (x-y)}{\sqrt{x y}},
\end{aligned}
$$

we can see that

$$
\left\langle j^{l}\right\rangle=\left\langle j^{l}\right\rangle_{0}+\frac{N e A_{p}}{V_{q}}(z / a)^{D+1} \sum_{\mathbf{n}_{q}} k_{l} \int_{k_{(q)}}^{\infty} d u \frac{\left(u^{2}-k_{(q)}^{2}\right)^{\frac{p-1}{2}}}{e^{2 u\left(z_{2}-z_{1}\right)}+1} .
$$

The massless fermionic field is conformally invariant and, as we could expect, the brane-induced contribution in (4.32) is conformally related to the corresponding expression for two parallel boundaries in the Minkowski bulk. The latter is obtained from (4.30) taking $m=0$.

Now let us consider the asymtotics for limiting cases of the brane locations. In the limit $z_{2} \rightarrow \infty$, for fixed $z_{1}$ and $z$, the right brane tends to the AdS horizon. In this limit, it is expected that from the results given above the current density will be obtained in the region $z_{1} \leq z<\infty$ for the geometry of a single brane at $z=z_{1}$. In order to show that we use the representation (4.16). The part with $\left\langle j^{l}\right\rangle^{(1)}$ does not depend on $z_{2}$ and it is sufficient to consider the limiting transition for the last term. The latter presents the contribution induced by the right brane. The dominant contribution comes from the region of the integration near the lower limit and from the mode in the summation over $\mathbf{n}_{q}$ with the smallest value of $k_{(q)}$. Under the assumption $\left|\tilde{\alpha}_{i}\right|<\pi$ that mode corresponds to $n_{i}=0$ for $i=p+1, \ldots, D-1$, and the corresponding value for $k_{(q)}$ is given by

$$
k_{(q)}^{(0) 2}=\sum_{i=p+1}^{D-1} \tilde{\alpha}_{i}^{2} / L_{i}^{2} .
$$

Hence, to the leading order, we get

$$
\begin{aligned}
\left\langle j^{l}\right\rangle \approx\left\langle j^{l}\right\rangle^{(1)}-\frac{\pi N e k_{(q)}^{(0)(p+1) / 2} \tilde{\alpha}_{l} z^{D+2} e^{-2 z_{2} k_{(q)}^{(0)}}}{2 V_{q} L_{l} a^{D+1}\left(4 \pi z_{2}\right)^{(p+1) / 2}} \\
\quad \times \sum_{j= \pm 1} j \frac{G_{\nu, \nu_{j}}^{2}\left(z_{1} k_{(q)}^{(0)}, z k_{(q)}^{(0)}\right)}{K_{\nu}^{2}\left(z_{1} k_{(q)}^{(0)}\right)} .
\end{aligned}
$$

This shows that when the right brane tends to the AdS horizon the corresponding contribution in the VEV of the current density is suppressed by the factor $e^{-2 z_{2} k_{(q)}^{(0)}} / z_{2}^{(p+1) / 2}$.

In the limit $z_{1} \rightarrow 0$, for fixed $z_{2}$ and $z$, the left brane tends to the AdS boundary. We use the representation (4.18), where the contribution of the left brane is given by the last term. To the leading order, that contribution is obtained by using the asymptotic expressions of the modified Bessel functions for small values of the arguments. In this way it can be seen that in the limit when the left brane tends to the AdS boundary the corresponding contribution to the current density vanishes as $z_{1}^{2 m a+1}$.

Now let us consider the asymptotics with respect to the lengths of compact dimensions. First let us discuss the case 
$L_{l} \ll L_{i}, i \neq l$. In this limit the contribution of the modes with large $\left|n_{i}\right|, i \neq l$, dominates in the VEV (4.17) and, to the leading order, the corresponding summations over $\mathbf{n}_{q-1}$ can be replaced by the integration:

$\sum_{\mathbf{n}_{q-1}} f\left(k_{(q-1)}\right) \rightarrow \frac{2(4 \pi)^{(1-q) / 2} V_{q}}{\Gamma((q-1) / 2) L_{l}} \int d x x^{q-2} f(x)$.

Next, we introduce a new integration variable $w=\sqrt{u^{2}-x^{2}-k_{l}^{2}}$ and then pass to polar coordinates in the $(x, w)$-plane. After integrating over the angular part one can see that, in the leading order, the current density is obtained in a $(D+1)$-dimensional model with a single compact dimension $x^{l}:\left.\left\langle j^{l}\right\rangle \approx\left\langle j^{l}\right\rangle\right|_{q=1}$. If additionally one has $L_{l} \ll z_{1}$, we can replace the modified Bessel functions by the corresponding asymptotic expressions for large arguments:

$$
\begin{aligned}
\left\langle j^{l}\right\rangle \approx & \left\langle j^{l}\right\rangle_{0}+\frac{2(4 \pi)^{(1-D) / 2} N e(z / a)^{D+1}}{\Gamma((D-1) / 2) L_{l}} \\
& \times \sum_{n_{l}=-\infty}^{+\infty} k_{l} \int_{\left|k_{l}\right|}^{\infty} d u \frac{\left(u^{2}-k_{l}^{2}\right)^{\frac{D-3}{2}}}{e^{2\left(z_{2}-z_{1}\right) u}+1}
\end{aligned}
$$

Comparing with (4.30), we see that the brane-induced contribution in (4.36) is conformally related to the corresponding current density for a massless fermionic field in $(D+1)$-dimensional Minkowski spacetime with a single compact dimension and with two planar boundaries having the distance $z_{2}-z_{1}$. In this limit the effects of the gravitational field are weak. Under the additional constraint $L_{l} \ll\left(z_{2}-z_{1}\right)$, the exponent in (4.36) is large and we can further simplify the corresponding expression. By taking into account that the dominant contribution comes from $k_{l}$ with the minimal value $\left|k_{l}\right|$, we get

$$
\left\langle j^{l}\right\rangle \approx\left\langle j^{l}\right\rangle_{0}+\frac{\pi^{(1-D) / 2} N e(z / a)^{D+1} \tilde{\alpha}_{l}\left|\tilde{\alpha}_{l}\right|^{\frac{D-3}{2}}}{2^{D-1} L_{l}^{(D+1) / 2}\left(z_{2}-z_{1}\right)^{(D-1) / 2}} e^{-2\left(z_{2}-z_{1}\right)\left|\tilde{\alpha}_{l}\right| / L_{l}},
$$

where it is assumed that $\left|\tilde{\alpha}_{l}\right|<\pi$. As seen, the braneinduced contribution is exponentially small. Note that in the same limit, $L_{l} \ll L_{i}, i \neq l$, and $L_{l} \ll z$, for the branefree contribution one has [25]

$$
\left\langle j^{l}\right\rangle_{0} \approx-\frac{e N L_{l} \Gamma((D+1) / 2)}{\pi^{(D+1) / 2}\left(a L_{l} / z\right)^{D+1}} \sum_{n_{l}=1}^{\infty} \frac{\sin \left(\tilde{\alpha}_{l} n_{l}\right)}{n_{l}^{D}} .
$$

and it dominates in the total current density.

For large values of $L_{l} \gg L_{i}, z_{1}, i \neq l$, it is more convenient to use the representation (4.23). The current density is dominated by the lowest mode for $\lambda_{n}$ and by the mode for which $k_{(q-1)}^{2}$ takes its minimal value. For $\left|\tilde{\alpha}_{i}\right|<\pi$ the latter corresponds to the mode with $n_{i}=0, i \neq l$, with the minimal value $k_{(q-1)}^{(0) 2}=\sum_{i=p+1, \neq l}^{D-1} \tilde{\alpha}_{i}^{2} / L_{i}^{2}$. By using the asymptotic expression of the Macdonald function for large arguments, we can see that in the limit under consideration the current density is suppressed by the factor $\exp \left[-L_{l} \sqrt{\lambda_{1}^{2} / z_{1}^{2}+k_{(q-1)}^{(0) 2}}\right]$.

If the one of the lengths $L_{i}, i \neq l$, is large compared to the other length scales in the model, the expression (4.17) for the current density is dominated by the terms with large values of $\left|n_{i}\right|$. To the leading order, we replace the corresponding summation by the integration and the VEV $\left\langle j^{l}\right\rangle$ coincides with the current density in the same model with decompactified $i$ th coordinate $x^{i}$. In the opposite limit of small $L_{i}, i \neq l$, assuming that $\left|\tilde{\alpha}_{i}\right|<\pi$, the behavior of the $l$ th component of the current density crucially depends on that wether $\tilde{\alpha}_{i}$ is zero or not. For $\tilde{\alpha}_{i}=0$ there is a zero mode along the $i$ th compact dimension with $n_{i}=0$ and it dominates in the current density $\left\langle j^{l}\right\rangle$. The leading term is obtained from (4.17) taking the contribution with $n_{i}=0$ and we get $\left\langle j^{l}\right\rangle \approx N z\left\langle j^{l}\right\rangle_{D} /\left(N_{D} a L_{i}\right)$, where $\left\langle j^{l}\right\rangle_{D}$ is the current density in the $D$-dimensional model with the absence of the $i$ th compact dimension, $N_{D}$ is the number of spinor components in that model. For $\tilde{\alpha}_{i} \neq 0$, again, the dominant contribution comes from the mode with $n_{i}=0$. The corresponding estimates can be done in a way similar to that for small values of $L_{l}$ and we can see that the braneinduced VEV is suppressed by the factor $e^{-2\left(z_{2}-z_{1}\right)\left|\tilde{\alpha}_{i}\right| / L_{i}}$.

In the numerical examples of this section we will consider the model $D=4$ with a single compact dimension $x^{D}$. For the corresponding values of the parameters one has $p=2$ and $q=1$. The length of the compact dimension will be denoted by $L$ and the corresponding phase by $\tilde{\alpha}$. Four different types of boundary conditions on the branes will be discussed (corresponding to roman numerals near the graphs). Graphs with I correspond to the bag boundary condition (2.5) and the graphs with II correspond to the condition (5.1) below. As it has been discussed in Sec. VI, depending on the parity of the field under the reflections with respect to the branes, two other classes of boundary condition may arise in $Z_{2}$-symmetric braneworld models. They correspond to the boundary conditions $Z_{m a+1 / 2}\left(\lambda z_{j}\right)=0$ (the graphs will be designated by III) and $Z_{m a-1 / 2}\left(\lambda z_{j}\right)=0$ (the graphs designated by IV) on both the branes $z=z_{j}, j=1,2$.

In Fig. 1 we have displayed the dependence of the current density on the phase $\tilde{\alpha}$. The current density is a periodic function of $\tilde{\alpha}$ and graphs are plotted for one period. For the parameters we have taken the values corresponding to $m a=1, z_{1} / L=0.5, z_{2} / L=1, z / L=0.75$. The dashed line corresponds to the current density in the geometry without branes. As seen, depending on the 


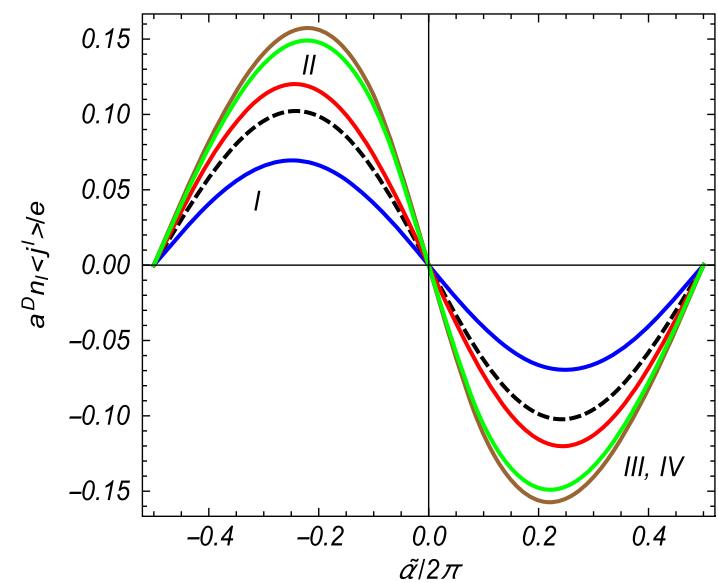

FIG. 1. Fermionic current along the compact dimension as a function of the phase in the periodicity condition in the model $D=4, p=2, q=1$. The roman numerals near the curves correspond to different classes of boundary conditions on the branes. The graphs are plotted for $z_{1} / L=0.5, z_{2} / L=1$, $z / L=0.75$, and $m a=1$.

boundary conditions imposed on the field, the presence of the branes can either increase or decrease the vacuum current density. In particular, the bag boundary condition reduces the current density.

The dependence of the current density on the field mass for different types of boundary conditions is presented in Fig. 2. For the phase in the periodicity condition we have taken $\tilde{\alpha}=\pi / 2$. The values of the remaining parameters are the same as those for Fig. 1. From the data in Fig. 2 we see that in the range of the mass $m a>1$ the brane-induced currents can be essentially larger compared with the currents in the branefree geometry. Of course, for $m a \gg 1$ both these contributions are suppressed. The coincidence of the current densities for a massless field in the cases of boundary conditions I,II and III,IV will be explained below on the base of the corresponding analytic expressions.

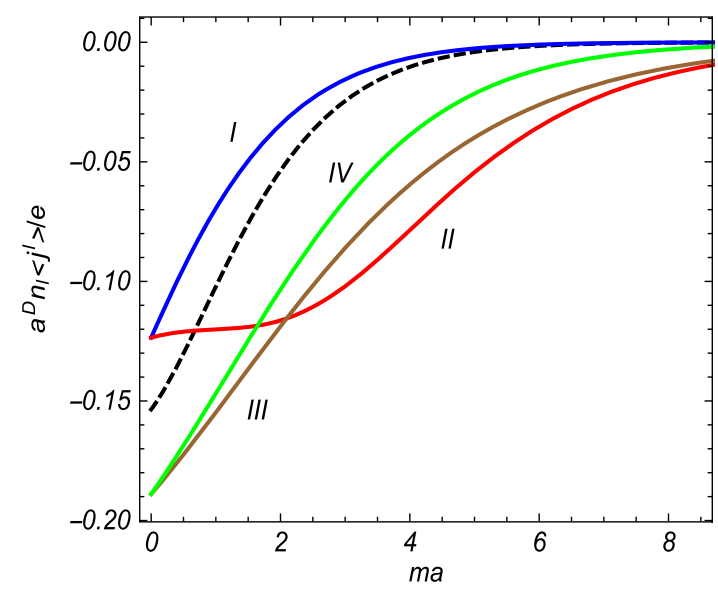

FIG. 2. The current density as a function of the field mass for $\tilde{\alpha}=\pi / 2$. The other parameters are the same as those for figure 1 .

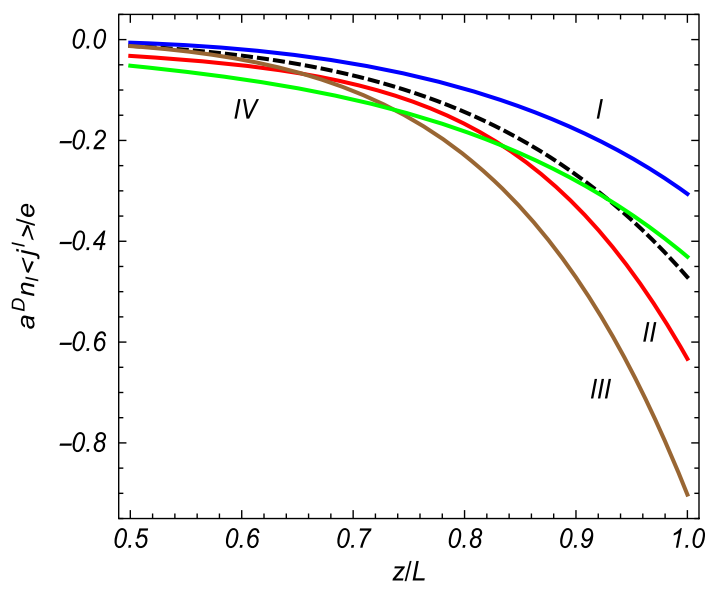

FIG. 3. The current density in the region between the branes as a function of the coordinate $z$ in units of the length of the compact dimension. The dashed curve presents the current density in the absence of the branes.

The behavior of the current density versus the coordinate $z$ is shown in Fig. 3 for $\tilde{\alpha}=\pi / 2, m a=1$ and for the locations of the branes we have taken $z_{1} / L=0.5$, $z_{2} / L=1$. As seen, the current density is mainly located near the right brane.

It is also of interest to consider the dependence of the current density on the length of the compact dimension. As it has been shown by the asymptotic analysis that dependence is essentially different for the branefree and brane-induced contributions. For small values of $L$ the branefree part behaves as $\left\langle j^{l}\right\rangle_{0} \propto 1 /(a L / z)^{D+1}$ and the brane-induced part is suppressed by the factor $e^{-2\left(z_{2}-z_{1}\right)|\tilde{\alpha}| / L}$ [see (4.37)]. This feature is seen in Fig. 4, where we have plotted the brane-induced current density, $\left\langle j^{l}\right\rangle_{b}=$ $\left\langle j^{l}\right\rangle-\left\langle j^{l}\right\rangle_{0}$, versus the ratio $L / z_{1}$ for $\tilde{\alpha}=\pi / 2, m a=1$, $z_{2} / z_{1}=2, z / z_{1}=1.5$.

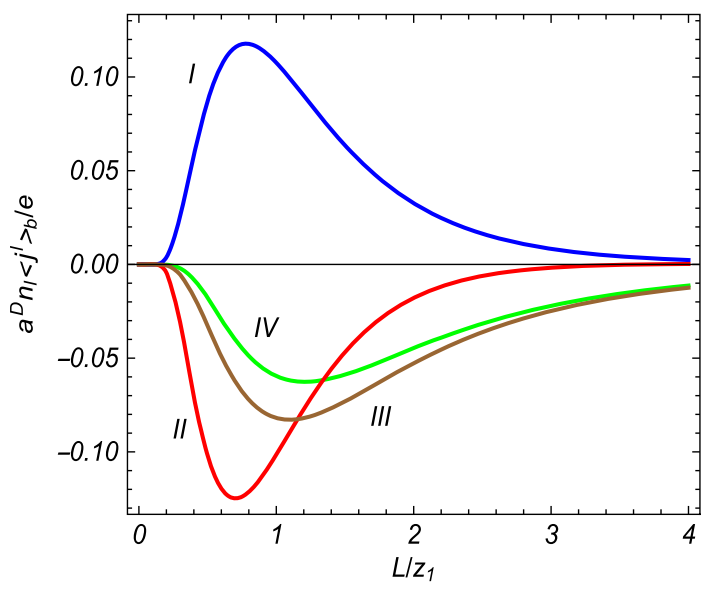

FIG. 4. The brane-induced contribution in the current density as a function of the length of the compact dimension. The graphs are plotted for $\tilde{\alpha}=\pi / 2, m a=1, z_{2} / z_{1}=2, z / z_{1}=1.5$. 


\section{SECOND CLASS OF BOUNDARY CONDITIONS}

For the normal $n_{\mu}^{(j)}$ to the brane at $z=z_{j}$ and for the Dirac matrices we have the relation $\left(i \gamma^{\mu} n_{\mu}^{(j)}\right)^{2}=1$. This means that $i \gamma^{\mu} n_{\mu}^{(j)}$ has eigenvalues \pm 1 . If we assume that on the brane $i \gamma^{\mu} n_{\mu}^{(j)} \psi= \pm \psi$ then for both the signs one gets $n_{\mu}^{(j)} j^{\mu}=0$ for $z=z_{j}$ and quantum numbers are not lost through the brane. In the discussion above we have considered the boundary condition with the lower sign. Equally well acceptable boundary condition is the one with the upper sign:

$$
\left(1-i \gamma^{\mu} n_{\mu}^{(j)}\right) \psi=0, \quad z=z_{j},
$$

with $j=1,2$. Both the boundary conditions (2.5) and (5.1) are compatible with self-adjointness of the Hamiltonian given by the Dirac operator. They are obtained considering a fermionic field interacting with a scalar potential outside the bounded region under consideration in the limit when the potential tends to infinity (see, for instance, Ref. [33]). The condition (2.5) has been employed in a phenomenological model, called the MIT bag model, to describe the confinement of quarks inside hadrons. Another physical realization of fermionic models with boundary conditions (2.5) and (5.1) is provided by electronic subsystems of graphene ribbons with zigzag types of cuts (see, e.g., [34]). As it will be discussed in Sec. VI, the boundary conditions of the type (2.5) and (5.1) appear also in $Z_{2}$-symmetric brane models. Note that the conditions (2.5) and (5.1) contain no additional parameters. More general boundary conditions for fermionic fields, ensuring the self-adjointness of the Hamiltonian and the zero normal projection of the current density at the boundary, have been discussed in Refs. [34,35]. Those boundary conditions involve additional parameters that encode the properties of the boundary. The corresponding applications include graphene ribbons with general cuts and chiral bag models of nucleons.

The positive and negative energy mode functions for the conditions (5.1) are still presented in the form (3.3). The boundary conditions on the left and right branes are reduced to the equations $Z_{m a-1 / 2}\left(\lambda z_{1}\right)=0$ and $Z_{m a+1 / 2}\left(\lambda z_{2}\right)=0$, respectively. Now the eigenvalues of the quantum number $\lambda$ are determined from the equation

$$
g_{\nu-1, \nu}\left(\lambda z_{1}, \lambda z_{2}\right)=0 .
$$

The corresponding positive roots with respect to $\lambda z_{1}$ will be denoted by $\lambda_{n}^{(-)}=\lambda z_{1}$. For large values of $\lambda$, by using the asymptotic expressions for the Bessel functions, to the leading order the equation (5.2) is reduced to $\cos \left[\lambda\left(z_{2}-z_{1}\right)\right]=0$. For a massless field this equation is exact. Hence, for large values of $n$ one has the asymptotic expression $\lambda_{n}^{(-)} \approx \pi(n-1 / 2) /\left(z_{2} / z_{1}-1\right)$. In the Minkowski bulk two problems with boundary conditions (2.5) and (5.1) differ by rearrangement of two planar boundaries and the VEVs in the region between the plates are the same. For the AdS bulk the boundaries have nonzero extrinsic curvature and that is not the case.

In a way similar to that we have described for the boundary conditions (2.5), for the mode functions one gets

$$
\begin{aligned}
\psi_{\beta}^{(+)}= & B_{\beta}^{(+)} z^{\frac{D+1}{2}} e^{i \mathbf{k} \mathbf{x}-i \omega t} \\
& \times\left(\begin{array}{c}
\frac{\mathbf{k} \chi \chi_{0}^{\dagger}+i \lambda-\omega}{\omega} g_{m a-1 / 2, m a+s / 2}\left(\lambda z_{1}, \lambda z\right) w^{(\sigma)} \\
i \chi_{0}^{\dagger} \frac{\mathbf{k} \chi \chi_{0}^{\dagger}+i \lambda+\omega}{\omega} g_{m a-1 / 2, m a-s / 2}\left(\lambda z_{1}, \lambda z\right) w^{(\sigma)}
\end{array}\right), \\
\psi_{\beta}^{(-)}= & B_{\beta}^{(-)} z^{\frac{D+1}{2}} e^{i \mathbf{k} \mathbf{x}+i \omega t} \\
& \times\left(\begin{array}{c}
i \chi_{0} \frac{\mathbf{k} \chi^{\dagger} \chi_{0}-i \lambda+\omega}{\omega} g_{m a-1 / 2, m a+s / 2}\left(\lambda z_{1}, \lambda z\right) w^{(\sigma)} \\
\frac{\mathbf{k} \chi^{\dagger} \chi_{0}-i \lambda-\omega}{\omega} g_{m a-1 / 2, m a-s / 2} \\
\omega
\end{array}\right),
\end{aligned}
$$

with the normalization coefficients

$$
\left|B_{\beta}^{( \pm)}\right|^{2}=\frac{(2 \pi)^{2-p} \lambda}{32 V_{q} a^{D} z_{1}} T_{m a-1 / 2}^{(-)}\left(\eta, \lambda_{n}^{(-)}\right)
$$

where

$$
T_{m a-1 / 2}^{(-)}(\eta, x)=x\left[\frac{J_{m a-1 / 2}^{2}(x)}{J_{m a+1 / 2}^{2}(\eta x)}-1\right]^{-1} .
$$

Likewise in the previous case the charge density and the components of the current density along uncompact dimensions vanish. The components along compact dimensions do not depend on the value of the parameter $s$ and are given by

$$
\begin{aligned}
\left\langle j^{l}\right\rangle= & -\frac{\pi^{2}(4 \pi)^{-p / 2} e N z^{D+2}}{4 \Gamma(p / 2) V_{q} a^{D+1} z_{1}} \sum_{\mathbf{n}_{q}} k_{l} \int_{0}^{\infty} d k_{(p)} k_{(p)}^{p-1} \\
& \times \sum_{n=1}^{\infty} \frac{\lambda_{n}^{(-)} T_{m a-1 / 2}^{(-)}\left(\eta, \lambda_{n}^{(-)}\right)}{\sqrt{\lambda_{n}^{(-) 2}+z_{1}^{2} k^{2}}} \\
& \times \sum_{j= \pm 1} g_{m a-1 / 2, m a+j / 2}^{2}\left(\lambda_{n}^{(-)}, \lambda_{n}^{(-)} z / z_{1}\right) .
\end{aligned}
$$

For a massless field the equation for the eigenvalues $\lambda_{n}^{(-)}$ coincides with that for $\lambda_{n}$ in the case of the bag boundary condition and the current densities coincide as well. The summation over the eigenmodes $\lambda_{n}^{(-)}$can be done by using the formula (A1) with $\mu=m a+1 / 2$ and $\delta=-1$. The part with the first term in the right-hand side of (A1) gives the contribution to the VEV from the left brane when the right one is absent. It is given by the expression 


$$
\left\langle j^{l}\right\rangle^{(1)}=\left\langle j^{l}\right\rangle^{(0)}-\frac{A_{p} e N z^{D+2}}{V_{q} a^{D+1}} \sum_{\mathbf{n}_{q}} k_{l} \int_{k_{(q)}}^{\infty} d u u\left(u^{2}-k_{(q)}^{2}\right)^{\frac{p-1}{2}} \frac{I_{m a-1 / 2}\left(z_{1} u\right)}{K_{m a-1 / 2}\left(z_{1} u\right)} \sum_{j= \pm 1} j K_{m a+j / 2}^{2}(z u) .
$$

The current density in the region between the branes is presented in the form

$$
\begin{aligned}
\left\langle j^{l}\right\rangle= & \left\langle j^{l}\right\rangle^{(0)}+\frac{A_{p} e N z^{D+2}}{V_{q} a^{D+1}} \sum_{\mathbf{n}_{q}} k_{l} \int_{k_{(q)}}^{\infty} d u u\left(u^{2}-k_{(q)}^{2}\right)^{\frac{p-1}{2}}\left[\frac{K_{\nu-1}\left(z_{1} u\right) I_{\nu}\left(z_{2} u\right)}{I_{\nu-1}\left(z_{1} u\right) K_{\nu}\left(z_{2} u\right)}+1\right]^{-1} \\
& \times \sum_{j= \pm 1} j\left[\frac{K_{\nu-1}\left(z_{1} u\right)}{I_{\nu-1}\left(z_{1} u\right)} I_{m a+j / 2}^{2}(z u)-\frac{I_{\nu}\left(z_{2} u\right)}{K_{\nu}\left(z_{2} u\right)} K_{m a+j / 2}^{2}(z u)+2 j I_{m a+j / 2}(z u) K_{m a+j / 2}(z u)\right] .
\end{aligned}
$$

An equivalent representations, similar to (4.16), (4.18), and (4.23), can also be obtained for the boundary condition (5.1). For the integrated current one gets the formula that is obtained from (4.27) with the replacement $\lambda_{n} \rightarrow \lambda_{n}^{(-)}$and the relation (4.28) remains the same.

Similar to (4.34), in the limit $z_{2} \rightarrow \infty$ the contribution of the second brane in the VEV of the current density is suppressed by the factor $e^{-2 z_{2} k_{(q)}^{(0)}}$. For the limit $z_{1} \rightarrow 0$ two cases should be considered separately. In the case $m a>1 / 2$ one gets

$$
\left\langle j^{l}\right\rangle \approx\left\langle j^{l}\right\rangle^{(2)}-\frac{2 A_{p} e N\left(z_{1} / 2\right)^{2 m a-1} z^{D+2}}{V_{q} a^{D+1} \Gamma(\nu-1) \Gamma(\nu)} \sum_{\mathbf{n}_{q}} k_{l} \int_{k_{(q)}}^{\infty} d u u^{2 m a}\left(u^{2}-k_{(q)}^{2}\right)^{\frac{p-1}{2}} \sum_{j= \pm 1} j \frac{G_{\nu, m a+j / 2}^{2}\left(z_{2} u, z u\right)}{I_{\nu}^{2}\left(z_{2} u\right)},
$$

where

$$
\left\langle j^{l}\right\rangle^{(2)}=\left\langle j^{l}\right\rangle^{(0)}+\frac{A_{p} e N z^{D+2}}{V_{q} a^{D+1}} \sum_{\mathbf{n}_{q}} k_{l} \int_{k_{(q)}}^{\infty} d u u\left(u^{2}-k_{(q)}^{2}\right)^{\frac{p-1}{2}} \sum_{j= \pm 1} j \frac{K_{\nu}\left(z_{2} u\right)}{I_{\nu}\left(z_{2} u\right)} I_{m a+j / 2}^{2}(z u) .
$$

In this case the contribution induced by the first brane tends to zero as $z_{1}^{2 m a-1}$. For $m a=1 / 2$ the corresponding decay is logarithmic, as $1 / \ln \left(z_{1} / z_{2}\right)$. For $m a<1 / 2$, to the leading order one gets

$$
\left\langle j^{l}\right\rangle \approx\left\langle j^{l}\right\rangle^{(2)}-\frac{2 A_{p} e N z^{D+2}}{\pi V_{q} a^{D+1}} \cos (m a \pi) \sum_{\mathbf{n}_{q}} k_{l} \int_{k_{(q)}}^{\infty} d u u \frac{\left(u^{2}-k_{(q)}^{2}\right)^{\frac{p-1}{2}}}{I_{\nu}\left(z_{2} u\right) I_{-\nu}\left(z_{2} u\right)} \sum_{j= \pm 1} j G_{\nu, m a+j / 2}^{2}\left(z_{2} u, z u\right) .
$$

This leading term is different from that in the first case [see (5.9)]. Note that if we consider a problem with the boundary condition (5.1) on the right brane but with the condition (2.5) on the left one, the limiting transition $z_{1} \rightarrow 0$ in the range of the field mass $m a<1 / 2$ is completely different, the left brane induced contribution behaves as $z_{1}^{2 m a+1}$. The last term in (5.11) for the boundary condition (5.1), in some sense, can be considered as a memory from the left brane when its location tends to the AdS boundary. This kind of memory is absent for the condition (2.5).

In Figs. 1-4, the curves corresponding to the boundary condition (5.1) are designated by II. As it has been mentioned above, in the case of a massless field the current densities for the boundary conditions (2.5) and (5.1) coincide. That is seen from Fig. 2. For a massive field the brane-induced contributions to the current density for the boundary condition (5.1) can be essentially larger when compared with the branefree part and the brane-induced part in the case of the condition (2.5).

\section{CURRENTS IN $Z_{2}$-SYMMETRIC MODELS WITH TWO BRANES}

With the results given above we can investigate the current density in higher dimensional generalizations of Randall-Sundrum type braneworlds [36] with two branes and with a compact subspace. In these models the coordinate $y$ is compactified on an orbifold $S^{1} / Z_{2}$ of length $b$, with $-b \leq y \leq b$. The branes are located at the points $y=0$ and $y=b$ and the line element is given by (2.1) where the warp factor $e^{-2 y / a}$ must be replaced by $e^{-2|y| / a}$. The original Randall-Sundrum model has a single extra dimension, corresponding to $D=4$, and only the gravitational field propagates on the bulk. However, in braneworld models motivated from string theories we expect the presence of extra compact dimensions and also extra bulk fields. Here we consider more general setup with the locations of the branes at $y=y_{j}, j=1,2$.

Among the motivations to consider bulk fields other than the graviton is related to the need for stabilization of the 
interbrane distance (radion field). The variations of the latter would imply the variations in physical constants on the visible brane. An example is the Goldberger-Wise stabilization mechanism [37], where the potential for the radion field is generated by a bulk scalar with quartic interactions localized on the branes. An alternative mechanism for the radion stabilization is based on the Casimir effect for bulk quantum fields. Another idea to consider bulk fields has been that the standard model fields are not strictly confined to branes, but merely localized around them. This approach to the braneworld concept is more universal and attractive from the point of view that gravitational field is not separated from other fields. However, it should be noted that even though we consider bulk fields other than the graviton, it will be assumed that the locally AdS geometry is not modified by those fields. This means that we neglect the backreaction of the bulk fields. Exact backreacted solutions are not available and the previous investigations of quantum effects in Randall-Sundrum braneworlds have been done under that assumption.

In braneworld models, the boundary conditions on the branes for bulk fields are obtained from the $Z_{2}$-symmetry. For a brane at $y=y_{j}$ and for a fermionic field $\psi(x)$ one has $\psi\left(x^{i}, y_{j}-y\right)=M_{j} \psi\left(x^{i}, y-y_{j}\right)$, where $M_{j}$ is a $N \times N$ matrix. From the invariance of the fermionic action under the $Z_{2}$ identification it can be seen that (see $[8,29]$ ) this matrix have the form

$$
M_{j}=-u_{j} s \operatorname{diag}(1,-1),
$$

where $u_{j}= \pm 1$ and we have extracted the factor $s$ for convenience. With this transformation matrix, the boundary condition for the modes (3.3) on the brane $y=y_{j}$ is reduced to $Z_{m a+u_{j} / 2}\left(\lambda z_{j}\right)=0$ for both the positive and negative energy solutions. We also see that with the choice (6.1) (opposite signs of the matrix $M$ for $s=1$ and $s=-1$ ), the boundary condition is the same for $s= \pm 1$. As a result, in the geometry of two branes one has four different combinations of the boundary conditions corresponding to different choices of $u_{j}$ in the set $\left(u_{1}, u_{2}\right)$ (for different combinations of boundary conditions imposed on fermionic fields in two-brane models see also [38]).

For given $\left(u_{1}, u_{2}\right)$, the mode functions obeying the boundary condition on the brane $y=y_{1}$ are presented as

$$
\begin{aligned}
\psi_{\beta}^{(+)}(x)= & D_{\beta}^{(+)} z^{\frac{D+1}{2}} e^{i \mathbf{k} \mathbf{x}-i \omega t} \\
& \times\left(\begin{array}{c}
\frac{\mathbf{k} \chi \chi_{0}^{\dagger}+i \lambda-\omega}{\omega} g_{m a+u_{1} / 2, m a+s / 2}\left(\lambda z_{1}, \lambda z\right) w^{(\sigma)} \\
i \chi_{0}^{\dagger} \frac{\mathbf{k} \chi \chi_{0}^{\dagger}+i \lambda+\omega}{\omega} g_{m a+u_{1} / 2, m a-s / 2}\left(\lambda z_{1}, \lambda z\right) w^{(\sigma)}
\end{array}\right), \\
\psi_{\beta}^{(-)}(x)= & D_{\beta}^{(-)} z^{\frac{D+1}{2}} e^{i \mathbf{k} \mathbf{x}+i \omega t} \\
& \times\left(\begin{array}{c}
i \chi_{0} \frac{\mathbf{k} \chi^{\dagger} \chi_{0}-i \lambda+\omega}{\omega} g_{m a+u_{1} / 2, m a+s / 2}\left(\lambda z_{1}, \lambda z\right) w^{(\sigma)} \\
\frac{\mathbf{k} \chi^{\dagger} \chi_{0}-i \lambda-\omega}{\omega} g_{m a+u_{1} / 2, m a-s / 2}\left(\lambda z_{1}, \lambda z\right) w^{(\sigma)}
\end{array}\right) .
\end{aligned}
$$

From the boundary condition on the brane $y=y_{2}$ it follows that now the eigenvalues for $\lambda$ are roots of the equation

$$
g_{m a+u_{1} / 2, m a+u_{2} / 2}\left(\lambda z_{1}, \lambda z_{2}\right)=0 .
$$

For the normalization coefficients we get

$$
\left|D_{\beta}^{( \pm)}\right|^{2}=\frac{\lambda T_{m a+u_{1} / 2}^{\left(u_{1}, u_{2}\right)}\left(z_{2} / z_{1}, \lambda z_{1}\right)}{32 N_{0} z_{1}(2 \pi)^{p-2} V_{q} a^{D}} .
$$

where

$$
T_{m a+u_{1} / 2}^{\left(u_{1}, u_{2}\right)}(\eta, x)=x\left[\frac{J_{m a+u_{1} / 2}^{2}(x)}{J_{m a+u_{2} / 2}^{2}(\eta x)}-1\right]^{-1} .
$$

Note that the Eq. (6.3) corresponds to the boundary conditions

$$
\left(1-(-1)^{j} i u_{j} \gamma^{\mu} n_{\mu}^{(j)}\right) \psi(x)=0, \quad z=z_{j},
$$

on the branes.

In $Z_{2}$-symmetric braneworld models the normalization integral goes over the two copies of the region $y_{1} \leq y \leq y_{2}$ and in (6.4) $N_{0}=2$. In the analog of the problem we have considered in the previous sections with two branes $y=y_{j}$ and with the boundary conditions $Z_{m a+u_{j} / 2}\left(\lambda z_{j}\right)=0$ on them, in the region $y_{1} \leq y \leq y_{2}$ one should take $N_{0}=1$ in (6.4). Note that for $u_{1}=u_{2}$ and for large values of $\lambda$ the Eq. (6.3) is reduced to $\sin \left[\lambda\left(z_{2}-z_{1}\right)\right]=0$ and for the corresponding modes one has asymptotic expression $\lambda z_{1}=\lambda_{m a+u_{1} / 2, n}^{(0)} \approx \pi n /\left(z_{2} / z_{1}-1\right)$ with large $n$. For massless fields this expression is exact.

Now we see that the current densities in $Z_{2}$-symmetric braneworlds with the combination of the boundary conditions on the branes corresponding to $\left(u_{1}, u_{2}\right)=(+1,-1)$ are obtained from the results in Sec. IV with an additional coefficient $1 / 2$. For the set of boundary conditions with $\left(u_{1}, u_{2}\right)=(-1,+1)$ the corresponding current density is obtained from the formulas in Sec. V (again, with the factor $1 / 2)$. The current densities for the combinations of the boundary conditions corresponding to $\left(u_{1}, u_{2}\right)=(+1,+1)$ and $\left(u_{1}, u_{2}\right)=(-1,-1)$ can be considered in a similar way we have described in Sec. IV for the case $\left(u_{1}, u_{2}\right)=(+1,-1)$. The VEV of the current density along the $l$ th compact dimension is presented in the form similar to (4.5), where now $\lambda$ is the root of the Eq. (6.3) with $u_{2}=u_{1}$. The summation formula for the series over these roots is obtained from (A1) with $\delta=0$ and $\mu=m a+u_{1} / 2$ and the further transformation for the VEV is similar to that in Sec. IV. The final expression for the current density in the region between the branes takes the form 


$$
\begin{aligned}
\left\langle j^{l}\right\rangle= & \left\langle j^{l}\right\rangle^{(0)}+\frac{u_{1} A_{p} e N z^{D+2}}{N_{0} V_{q} a^{D+1}} \sum_{\mathbf{n}_{q}} k_{l} \int_{k_{(q)}}^{\infty} d u u\left(u^{2}-k_{(q)}^{2}\right)^{\frac{p-1}{2}}\left[\frac{K_{\mu}\left(z_{1} u\right) I_{\mu}\left(z_{2} u\right)}{K_{\mu}\left(z_{2} u\right) I_{\mu}\left(z_{1} u\right)}-1\right]^{-1} \\
& \times \sum_{j= \pm 1} j\left[\frac{I_{\mu}\left(z_{2} u\right)}{K_{\mu}\left(z_{2} u\right)} K_{m a+j / 2}^{2}(z u)+\frac{K_{\mu}\left(z_{1} u\right)}{I_{\mu}\left(z_{1} u\right)} I_{m a+j / 2}^{2}(z u)-2 j u_{1} I_{m a+j / 2}(z u) K_{m a+j / 2}(z u)\right],
\end{aligned}
$$

where $\mu=m a+u_{1} / 2$ and $u_{1}= \pm 1$. The single brane contribution to the vacuum current density for the brane at $y=y_{1}$ is given by (4.12) for $u_{1}=1$ and by the last term in (5.7) for $u_{1}=-1$ (with additional factors $1 / N_{0}$ for $Z_{2}$-symmetric braneworlds). We can also obtain an alternative representation similar to (4.23).

In Figs. 1-4, the graphs for the current densities (6.7) (with $N_{0}=1$ ) in the cases $u_{1}=u_{2}=+1$ and $u_{1}=u_{2}=$ -1 are designated by roman numerals III and IV, respectively. Note that one has the relation $g_{-\mu,-\mu}(x, u)=$ $g_{\mu, \mu}(x, u)$ and the eigenmodes for $\lambda$ in these cases coincide for a massless field. From here it follows that the current densities corresponding to III and IV are the same in the limit $m \rightarrow 0$. This is seen from Fig. 2 .

In the Randall-Sundrum scenario the standard model fields are localized on the brane $z=z_{2}$ (visible or infrared brane). The current density on that brane is a source of magnetic fields having components in the uncompact subspace as well. It is of interest to separate the parts in the current density on the visible brane induced by the presence of the hidden (or ultraviolet) brane with the location $z=z_{1}$. By using the expressions given above, we can combine the hidden brane-induced contributions for different combinations of the boundary conditions, specified by the set $\left(u_{1}, u_{2}\right)$, in a single expression

$$
\begin{aligned}
{\left[\left\langle j^{l}\right\rangle-\left\langle j^{l}\right\rangle^{(2)}\right]_{z=z_{2}}=} & \frac{A_{p} e N z_{2}^{D}}{N_{0} V_{q} a^{D+1}} \sum_{\mathbf{n}_{q}} k_{l} \int_{k_{(q)}}^{\infty} d u \frac{I_{m a+u_{1} / 2}\left(z_{1} u\right)}{u I_{m a+u_{2} / 2}\left(z_{2} u\right)} \\
& \times \frac{\left(u^{2}-k_{(q)}^{2}\right)^{\frac{p-1}{2}}}{G_{m a+u_{1} / 2, m a+u_{2} / 2}\left(z_{1} u, z_{2} u\right)}
\end{aligned}
$$

In braneworld models of the Randall-Sundrum type, in order to solve the hierarchy problem between the Planck and electroweak energy scales, it is assumed that $\left(y_{2}-y_{1}\right) \gg a$. Under this condition one has $z_{2} / z_{1} \gg 1$ and the asymptotic behavior of (6.8) depends on the lengths of compact dimensions. For $z_{1} / L_{i} \gtrsim 1$, in the integration range of (6.8) one has $z_{2} x / z_{1} \gg 1$. In a way similar to that we have used for (4.34), it can be seen that to the leading order one has

$$
\begin{aligned}
{\left[\left\langle j^{l}\right\rangle-\left\langle j^{l}\right\rangle^{(2)}\right]_{z=z_{2}} \approx } & -\frac{e N z_{2}^{q+2} \tilde{\alpha}_{l}\left(z_{2} k_{(q)}^{(0)}\right)^{(p-1) / 2}}{2^{p+1} \pi^{(p-1) / 2} N_{0} V_{q} L_{l} a^{D+1}} \\
& \times \frac{I_{m a+u_{1} / 2}\left(z_{1} k_{(q)}^{(0)}\right)}{K_{m a+u_{1} / 2}\left(z_{1} k_{(q)}^{(0)}\right)} e^{-2 z_{2} k_{(q)}^{(0)}},
\end{aligned}
$$

where $k_{(q)}^{(0)}$ is defined by (4.33). For $z_{2} / L_{i} \lesssim 1$ and $z_{2} / z_{1} \gg 1$ the asymptotic expression of (6.8) is found in a way similar to that used above for the limit $z_{1} \rightarrow 0$. For $m a+u_{1} / 2>0$ we can see that the hidden brane contribution in the current density on the visible brane behaves like $\left(z_{1} / z_{2}\right)^{2 m a+u_{1}}$. In the case $m a+u_{1} / 2<0$ (for non-negative $m$ this implies $\left.u_{1}=-1\right)$ one gets

$$
\begin{aligned}
& {\left[\left\langle j^{l}\right\rangle-\left\langle j^{l}\right\rangle^{(2)}\right]_{z=z_{2}}} \\
& \approx \frac{2 u_{2} A_{p} e N z_{2}^{q+2}}{\pi N_{0} V_{q} a^{D+1}} \sum_{\mathbf{n}_{q}} k_{l} \int_{z_{2} k_{(q)}}^{\infty} d x \frac{\cos (\pi m a)\left(x^{2}-z_{2} k_{(q)}^{2}\right)^{\frac{p-1}{2}}}{x I_{m a+u_{2} / 2}(x) I_{-m a-u_{2} / 2}(x)},
\end{aligned}
$$

and the leading term does not depend on $z_{1}$.

The discussion given above shows that the physical characteristics of the vacuum state, such as the VEVs of the charge and current densities, crucially depend on the phases $\tilde{\alpha}_{l}$. Another interesting physical effect related to these phases is the dynamical mass generation by compact extra dimensions (the so called Hosotani mechanism) [39]. In the setup we consider, the phases $\tilde{\alpha}_{l}$ are external parameters and their values are not fixed. Those values can be fixed dynamically by taking into account that the vacuum energy will also depend on the parameters $\tilde{\alpha}_{l}$ and the equilibrium values will correspond to the minimum of the effective potential. This issue has been discussed in the literature for the Minkowskian spacetime as the uncompact subspace (see, e.g., [40] and references therein). Having the complete set of fermionic modes, the evaluation of the vacuum energy density and the effective potential in the problem at hand can be done by applying the formula (A1) to the corresponding mode sums, in a way similar to that we have described for the charge and current densities. This requires a separate consideration and will be presented elsewhere.

\section{P- AND T-REVERSAL SYMMETRIC ODD-DIMENSIONAL MODELS AND APPLICATIONS TO CURVED GRAPHENE TUBES}

In this section we consider features of fermionic models in odd-dimensional spacetimes. As it has been already mentioned, for even $D$ there are two inequivalent irreducible representations of the Clifford algebra. For flat spacetime Dirac matrices $\gamma^{(b)}$ with $b=0, \ldots, D-1$, we introduce the $2^{D / 2} \times 2^{D / 2}$ matrix $\gamma=\prod_{b=0}^{D-1} \gamma^{(b)}$. Then we can 
take the matrix $\gamma^{(D)}$ in the form $\gamma_{(s)}^{(D)}=s \gamma$ for $D=4 n$ and in the form $\gamma_{(s)}^{(D)}=s i \gamma$ for $D=4 n-2$, where $n=1,2, \ldots$. Here, $s=+1$ and $s=-1$ correspond to two irreducible representations of the Clifford algebra. For the curved spacetime matrix $\gamma_{(s)}^{D}$, corresponding to the geometry described by (2.1), one can take $\gamma_{(s)}^{D}=(z / a) \gamma_{(s)}^{(D)}$. Let us denote by $\psi_{(s)}$ the fermioinc field realizing the representations with given $s$. The mass term in the corresponding Lagrangian density $\mathcal{L}_{(s)}=\bar{\psi}_{(s)}\left[i \gamma_{(s)}^{\mu}\left(\partial_{\mu}+\Gamma_{\mu}^{(s)}\right)-m\right] \psi_{(s)}$, with the set of Dirac matrices $\gamma_{(s)}^{\mu}=\left(\gamma^{0}, \gamma^{1}, \ldots \gamma^{D-1}, \gamma_{(s)}^{D}\right)$ and the related spin connection $\Gamma_{\mu}^{(s)}$, is not invariant under the charge conjugation $(C)$ and parity transformation $(P)$ in spatial dimensions $D=4 n$, and under the $P$-transformation and the time reversal $(T)$ in $D=4 n+2$.

We can construct fermionic models in odd-dimensional spacetimes, invariant under the $C$-, $P$ - and $T$-transformations, combining two fields $\psi_{(s)}$ with the Lagrangian density $\mathcal{L}=$ $\sum_{s= \pm 1} \mathcal{L}_{(s)}$. By appropriate transformations of the fields one can make this combined Lagrangian density invariant under the $C_{-}, P_{-}$, and $T$-transformations. Introducing $2 N \times$ $2 N$ matrices $\gamma^{(2 N) \mu}=\operatorname{diag}\left(\gamma_{(+1)}^{\mu}, \gamma_{(-1)}^{\mu}\right)$, with $N=2^{D / 2}$, and the corresponding spin connection $\Gamma_{\mu}^{(2 N)}$, we can combine two fields $\psi_{(s)}$ in a single $2 N$-component field $\Psi=$ $\left(\psi_{(+1)}, \psi_{(-1)}\right)^{T}$ with the Lagrangian density

$$
\mathcal{L}=\bar{\Psi}\left[i \gamma^{(2 N) \mu}\left(\partial_{\mu}+\Gamma_{\mu}^{(2 N)}\right)-m\right] \Psi
$$

and the current density operator $J^{\mu}=e \bar{\Psi} \gamma^{(2 N) \mu} \Psi$. An alternative representation of the model with two fields is obtained by making the field transformations $\psi_{(+1)}^{\prime}=$ $\psi_{(+1)}, \psi_{(-1)}^{\prime}=\gamma \psi_{(-1)}$. The combined Lagrangian density is presented as $\mathcal{L}=\sum_{s= \pm 1} \bar{\psi}_{(s)}^{\prime}\left[i \gamma^{\mu}\left(\partial_{\mu}+\Gamma_{\mu}\right)-s m\right] \psi_{(s)}^{\prime}$, where $\Gamma_{\mu}$ is the spin connection for the set of Dirac matrices $\gamma^{\mu}=\gamma_{(+1)}^{\mu}$. In this representation the Lagrangian densities for the fields with $s=+1$ and $s=-1$ differ by the sign of the mass term.

In the system of two fermionic fields $\psi_{(s)}$ the VEV of the current density is the sum of the VEVs coming from the separate fields $\left\langle J^{\mu}\right\rangle=\sum_{s= \pm 1}\left\langle j_{(s)}^{\mu}\right\rangle$. As we have seen above, if the boundary and periodicity conditions for the fields $\psi_{(s)}$ are the same, then the separate contributions $\left\langle j_{(s)}^{\mu}\right\rangle$ are the same as well and the total current density is obtained from the expressions given above with an additional factor 2 . However, both the boundary conditions and the phases in the periodicity conditions can be different for $s=+1$ and $s=-1$. In particular, we can combine various boundary conditions of the form (6.6) with different values of the parameters $u_{j}$ for separate fields. The corresponding VEVs for the current density for $s=+1$ and $s=-1$ are obtained from the formulas given above. An example of a condensed matter realization of the problem with different phases in the periodicity conditions for the fields $\psi_{(+1)}$ and $\psi_{(-1)}$ is provided by semiconducting carbon nanotubes (see below).

Among the most important applications of $D=2$ fermionic models are the so called Dirac materials. They include graphene, topological insulators and Weyl semimetals. For these materials the long-wavelength excitations of the electronic subsystem are well described by the Dirac equation with the velocity of light replaced by the Fermi velocity $v_{F}$. Here we specify the consideration for graphene. For a given quantum number $S= \pm 1$, corresponding to spin degrees of freedom, the analog of the Lagrangian density (7.1) with $N=2$ is written for a 4-component spinor field $\Psi_{S}=\left(\psi_{+, A S}, \psi_{+, B S}, \psi_{-, A S}\right.$, $\left.\psi_{-, B S}\right)^{T}$. Here, the indices + and - correspond to two inequivalent Fermi points at the corners of the Brillouin zone (points $\mathbf{K}_{+}$and $\mathbf{K}_{-}$) and the indices $A$ and $B$ correspond to the triangular sublattices of the graphene hexagonal lattice. The separate components of $\Psi_{S}$ present the corresponding amplitude of the electron wave function (see, for example, [41]). For the fields we have introduced before one has $\psi_{( \pm 1)}=\left(\psi_{ \pm, A S}, \psi_{ \pm, B S}\right)^{T}$. The mass term in the Dirac equation is expressed in terms of the energy gap $\Delta$ by the relation $m=\Delta / v_{F}^{2}$. This gap can be generated by a number of mechanisms. For the corresponding Compton wavelength one has $a_{C}=\hbar v_{F} / \Delta$.

The graphene is an interesting arena for investigation of various kinds of topological effects in field theory (for topological effects in condensed matter physics see, for example, [42]). The graphene made structures with nontrivial topology include fullerens, carbon nanotubes and nanoloops, and graphitic cones. They all have been experimentally observed. The spatial topology of the problem with $D=2$, we have considered above, corresponds to that for carbon nanotubes (topology $S^{1} \times R^{1}$ ). In graphene nanotubes the phases in the periodicity conditions (2.3) for the fields $\psi_{(s)}$ depend on the chirality of the tube. For metallic nanotubes one has $\alpha_{1} \equiv \alpha=0$ for both the fields $s=+1$ and $s=-1$. For semiconducting nanotubes the phases have opposite signs for spinors corresponding to the points $\mathbf{K}_{ \pm}$and $\alpha= \pm 2 \pi / 3$.

For a cylindrical nanotube rolled-up from a planar graphene sheet the spacetime geometry is flat. The corresponding VEV of the fermionic current density induced by the threading magnetic flux has been discussed in [16] for infinite length tubes and in [18] for finite length tubes. For the problem under consideration in the present paper, the spatial geometry, written in terms of the angular coordinate $\varphi=2 \pi x^{1} / L, 0 \leq \varphi \leq 2 \pi$, is given by the line element $d l^{2}=d y^{2}+(L / 2 \pi)^{2} e^{-2 y / a} d \varphi^{2}$ with $y_{1} \leq y \leq y_{2}$. This describes a finite length curved circular tube with the radius $r=L e^{-y / a} / 2 \pi$ depending on the coordinate along 


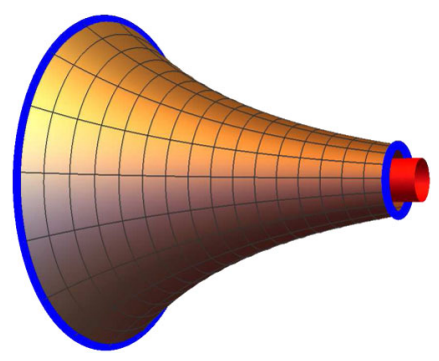

FIG. 5. The $D=2$ spatial geometry with two edges embedded in $R^{3}$.

the tube axis (for the curvature effects in graphene structures see also $[43,44])$. The corresponding 2-dimensional surface with two edges, embedded in 3-dimensional Euclidean space, is depicted in Fig. 5. In the figure we have also shown the magnetic flux enclosed by the curved tube. The graphene tubes with spatial geometry described by the line element $d l^{2}$ have been discussed in [44]. The geometry corresponds to Beltrami pseudosphere with Gaussian curvature $-1 / a^{2}$. The generation of a pseudosphere configurations from a planar graphene sheet has been recently discussed in [45] (see also the references therein). The corresponding curvature radius varies in the range $1.5 \mathrm{~nm}<a<74 \mathrm{~nm}$. Examples of wormhole geometries realized by curved graphene sheets have been considered in [46]. An important difference in the geometry we consider is that $g_{00}=e^{-2 y / a} \neq 1$. A number of mechanisms have been discussed recently for generation of the nontrivial $g_{00}$-component of the metric tensor for the low-energy effective field theory describing the dynamics of electrons in graphene. This can be done by various types of external fields, by deformations of graphene lattice (strains), and by the local variations in the Fermi velocity (for reviews see [47]).

In graphene tubes with the geometry under consideration the current density for a given spin $S$ is obtained by summing the contributions $\left\langle j_{(s)}^{\mu}\right\rangle$ coming from the fields $\psi_{(s)}$ corresponding to the points $\mathbf{K}_{ \pm}$. In the expression for the corresponding operator for spatial components an additional factor $v_{F}$ should be added, $j_{(s)}^{\mu}=e v_{F} \bar{\psi} \gamma_{(s)}^{\mu} \psi$ (with $e=-|e|$ for electrons). The expressions for $\left\langle j_{(s)}^{\mu}\right\rangle$ are obtained from the formulas given above taking $D=2$, $p=0, q=1$. We can also express the product $m a$ in terms of the Compton wavelength corresponding to the energy gap as $m a=a / a_{C}$. In the absence of the magnetic flux, the VEV of the current density vanishes in both metallic and semiconducting graphene tubes. In metallic tubes the separate contributions $\left\langle j_{(s)}^{\mu}\right\rangle$ are zero, whereas in semiconducting tubes $\left\langle j_{(-1)}^{\mu}\right\rangle=-\left\langle j_{(+1)}^{\mu}\right\rangle$ (assuming that the boundary conditions on the edges of the tube are the same for separate fields) because of the opposite signs of the phases in the periodicity conditions. Nonzero net currents may appear in the presence of the magnetic flux enclosed by the tube. In the absence of the magnetic flux, nonzero ground state currents in semiconducting tubes can be alternatively generated by imposing different boundary conditions on the edges for separate fields $\psi_{(+1)}$ and $\psi_{(-1)}$.

In Fig. 6 we have plotted the edge contribution in the fermionic current density, $\left\langle J^{\mu}\right\rangle_{b}=\left\langle J^{\mu}\right\rangle-\left\langle J^{\mu}\right\rangle_{0}$, in semiconducting tubes as a function of the enclosed magnetic flux (in units of flux quantum). The total current density (for a given $S$ ) is obtained summing the current densities for the fields $\psi_{(+1)}$ and $\psi_{(-1)}$ with the phases in the periodicity condition $2 \pi / 3$ and $-2 \pi / 3$, respectively. The left and right panels correspond to the boundary conditions (2.5) and (5.1), respectively. The graphs are plotted for $L / z_{1}=0.5$, $0.75,1$ (the numbers near the curves) and for fixed $a / a_{C}=1, z_{2} / z_{1}=2, z / z_{1}=1.5$.

In Fig. 7 the edge-induced current density is displayed as a function of the tube coordinate circumference for semiconducting nanotube. The curves I and II correspond to the boundary conditions (2.5) and (5.1), respectively, for both

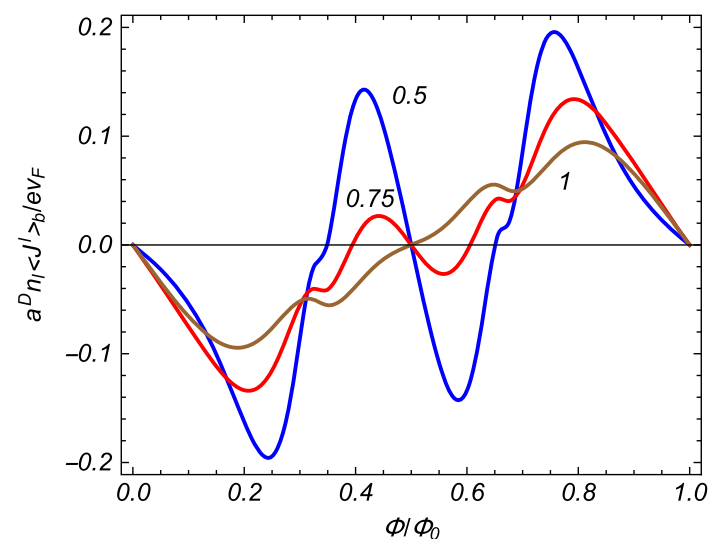

FIG. 6. The edge-induced current density as a function of the magnetic flux for semiconducting nanotubes. The left and right panels correspond to the boundary conditions (2.5) and (5.1). The graphs are plotted for $a / a_{C}=1, z_{2} / z_{1}=2, z / z_{1}=1.5$ and the numbers near the curves are the values of the ratio $L / z_{1}$. 


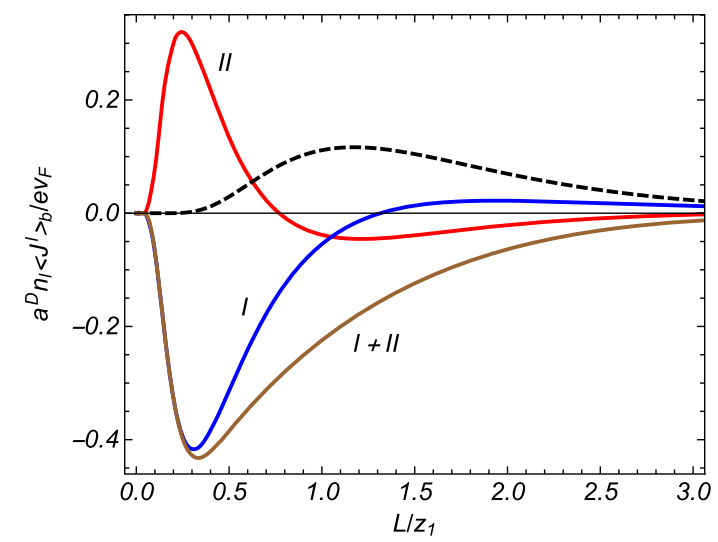

FIG. 7. The edge-induced current density as a function of the tube coordinate circumference for curved semiconducting nanotubes. The graphs are plotted for different choices of the boundary conditions on the tube edges. For the values of the related parameters see the text.

the fields $\psi_{(+1)}$ and $\psi_{(-1)}$. The curve I + II and the dashed curve correspond to the situation when the boundary condition (2.5) is imposed for the field $\psi_{(+1)}$ and the boundary condition (5.1) for the field $\psi_{(-1)}$. The graphs I, II, I + II are plotted for the magnetic flux $\Phi=0.4 \Phi_{0}$ and the dashed graph corresponds to $\Phi=0$. For the values of the remaining parameters we have taken $a / a_{C}=1$, $z_{2} / z_{1}=2, z / z_{1}=1.5$. The dashed curve in Fig. 7 presents an example where a nonzero current density is generated in the absence of magnetic flux by imposing different boundary conditions on separate fields corresponding to different Fermi points.

Note that we have considered a model where the only interaction of the fermionic field is with background classical gravitational and electromagnetic fields. The effects of geometry, topology and boundaries in models with four-fermion interactions have been discussed in [48]. The geometrical and topological aspects of electronic interactions in graphene and related materials are reviewed in [49].

\section{CONCLUSION}

Among the most important local characteristics of the vacuum state for charged fields is the VEV of the current density. We have studied the effects of two parallel branes on the current density in locally AdS spacetime with a part of spatial dimensions (in Poincaré coordinates) compactified to a torus. Along compact dimensions quasiperiodicity conditions were imposed with general values of the phases and the presence of a constant gauge field is assumed. The influence of the latter on the physical properties of the vacuum state is of Aharonov-Bohm type and is related to the nontrivial topology of the background geometry. On the branes we have considered several types of boundary conditions including the ones arising in $Z_{2}$-symmetric braneworld models. In the region between the branes, the eigenvalues of the radial quantum number are zeros of the combinations of the Bessel and Neumann functions. The mode sum for the VEV of the current density contains series over those eigenvalues. In order to find an integral representation, convenient in numerical calculations, we have used a variant of the generalized Abel-Plana formula that allowed to extract explicitly the brane-induced contributions. For all the boundary conditions discussed, the VEVs of the charge density and of the components of the current density along uncompact dimensions vanish.

In the investigation of the current density along compact dimensions, first we have considered the case of the bag boundary condition that is the most frequently one used for confinement of fermionic fields. In the region between the branes the $l$ th component is presented as (4.17) where the brane-induced contribution is explicitly extracted. The vacuum currents in the absence of the branes were investigated in [25] and here we were mainly concerned about the brane-induced effects. We have also provided representations, given by (4.8) and (4.18), with the separated contribution of the second brane when one adds it to the configuration with a single brane. The effects of the phases in the periodicity conditions and of the gauge field are encoded in the parameters $\tilde{\alpha}_{i}$. All the contributions to the $l$ th component of the current density are odd periodic functions of $\tilde{\alpha}_{l}$ and even periodic functions of $\tilde{\alpha}_{i}, i \neq l$, with the period $2 \pi$. In terms of the magnetic flux enclosed by the compact dimension, this correspond to the periodicity with the period equal to the flux quantum. An alternative representation of the current density, well adapted for the investigation of the near-brane asymptotic, is given by (4.23). Unlike to the initial representation (4.5), the series over the eigenvalues of the radial quantum number is exponentially convergent. The new representation also explicitly shows the finiteness of the current density on the branes. The latter feature is in clear contrast to the onbrane behavior of the fermion condensate and of the VEV of the energy-momentum tensor having surface divergences. The current density, integrated over the region between the branes, is connected to the on-brane values of the current density by a simple relation (4.28).

The general expression for the current density is rather complicated and, in order to clarify its behavior as a function of the parameters, we have considered various asymptotic limits. First of all, in the limit of large curvature radius the result is obtained for the geometry of two parallel plates in a locally Minkowski spacetime with a toroidal subspace, previously discussed in [18]. For a massless fermionic field, the problem under consideration is conformally related to the corresponding problem in locally Minkowski bulk and the current density is given by a simple expression (4.32). In the limit when the right brane tends to the AdS horizon, for fixed location of the left brane and of the observation point, the corresponding 
contribution to the current density is exponentially suppressed by the factor $e^{-2 z_{2} k_{(q)}^{(0)}}$, with $k_{(q)}^{(0)}$ defined by (4.33). When the location of the left brane tends to the AdS boundary, the corresponding contribution to the vacuum current decays like $z_{1}^{2 m a+1}$.

If the length of the $l$ th compact dimension $L_{l}$ is much smaller than the other length scales in the problem, including the difference $z_{2}-z_{1}$, the brane-induced contribution to the current density along that direction is suppressed by the factor $\exp \left[-2\left(z_{2}-z_{1}\right)\left|\tilde{\alpha}_{l}\right| / L_{l}\right]$ and the total current is dominated by the brane-free part. For large values of $L_{l}$, the current density is dominated by the mode with the lowest value $\lambda=\lambda_{1} / z_{1}$ of the radial quantum number and the current density is suppressed by the factor $\exp \left[-L_{l} \sqrt{\lambda_{1}^{2} / z_{1}^{2}+k_{(q-1)}^{(0) 2}}\right]$. The behavior of the lth component of the current density for small values of the length $L_{i}$, $i \neq l$, crucially depends whether the phase $\tilde{\alpha}_{i},\left|\tilde{\alpha}_{i}\right|<\pi$, is zero or not. For $\tilde{\alpha}_{i}=0$ the dominant contribution comes from the zero mode along the $i$ th dimensions and, to the leading order, the current density $\left\langle j^{l}\right\rangle$ is expressed in terms of the corresponding current density in $D$-dimensional spacetime with excluded $i$ th dimension. In the case $\tilde{\alpha}_{i} \neq 0$, the $\operatorname{VEV~}\left\langle j^{l}\right\rangle$ is suppressed by the factor $e^{-2\left(z_{2}-z_{1}\right)\left|\tilde{\alpha}_{i}\right| / L_{i}}$.

The investigation of the current density for the boundary condition (5.1) is done in a way similar to that in the case of the bag boundary condition. The corresponding current density in the region between the branes is decomposed as (5.8). For the mass range $m a<1 / 2$, an important difference when compared to the bag boundary conditions appears in the limit when the left brane goes to the AdS boundary $\left(z_{1} \rightarrow 0\right)$. An additional contribution survives (last term in (5.11)) that can be interpreted as some kind of memory from the boundary condition we have imposed on the brane at $z=z_{1}$. Yet another two classes of boundary conditions arise in $Z_{2}$-symmetric braneworld models. They correspond to the sets $\left(u_{1}, u_{2}\right)$ with $u_{1}=u_{2}$ in the conditions (6.6). The corresponding current densities are given by (6.7) with $\mu=m a+u_{1} / 2$. The memory effect in the limit $z_{1} \rightarrow 0$ is present for the boundary condition with $u_{1}=-1$. Depending on the boundary conditions imposed, the presence of the branes can either increase or decrease the current density. In braneworld models of the RandallSundrum type the observers are localized on the right brane and it is of interest to investigate the effects of the hidden brane on the current density on the visible brane. The part of the vacuum current induced by the hidden brane is given by (6.8). For the solution of the hierarchy problem between the electroweak and Planck energy scales it is required to have $z_{2} / z_{1} \gg 1$. In this limit the behavior of the hidden brane-induced current essentially depends on the lengths of compact dimensions and is different for $z_{1} / L_{i} \gtrsim 1$ and $z_{2} / L_{i} \lesssim 1$.

In odd-dimensional spacetimes, the models with massive fermionic fields realizing irreducible representations of the
Clifford algebra are not parity and time-reversal invariant. Fermionic models with parity and time-reversal symmetry are constructed combining two fields corresponding to inequivalent representations. If the periodicity conditions along compact dimensions and the boundary conditions on the branes are the same for separate fields, when the current densities for those fields are the same as well and the expressions for the total current density is obtained from those presented with an additional factor two. However, both the periodicity and boundary conditions can be different for fields realizing inequivalent representations of the Clifford algebra. An example of $D=2$ fermionic system with that type of situation is provided by semiconducting carbon nanotubes, with the electronic subsystem described by the Dirac model. In the corresponding setup the phases for separate fields have opposite signs and, in the absence of the magnetic flux, the corresponding current densities cancel each other if the boundary conditions for the fields are the same. In the case of different boundary conditions on the tube edges for separate fields, a nonzero current can be generated in the absence of magnetic flux. Curved graphene structures provide an important laboratory for the investigation of curvature and topological effects in quantum field theory. The special case $D=2$ of our model presents an exactly solvable problem of that kind.

\section{ACKNOWLEDGMENTS}

A. A. S. was supported by Viktor Ambartsumian Research Fellowship 2019-2020. A. A. S. gratefully acknowledges the hospitality of the INFN, Laboratori Nazionali di Frascati (Frascati, Italy), where a part of this work was done. V. V. V. was supported by De Sitter cosmology fellowship and by the Grant No. 18T-1C355 of the Committee of Science of the Ministry of Education and Science RA.

\section{APPENDIX: SUMMATION FORMULA OVER THE ZEROS OF COMBINATIONS OF CYLINDER FUNCTIONS}

In this Appendix we derive a summation formula over the positive zeros $x=\lambda_{\mu, n}^{(\delta)}, n=1,2, \ldots$, of the function $g_{\mu+\delta, \mu}(x, \eta x)$, with $\delta=0, \pm 1$ and $\eta>1$, by using the more general result from [50,51]. Note that the equation $g_{\mu+\delta, \mu}(x, \eta x)=0$ includes the equations for the eigenvalues of the radial quantum number $\lambda$ for the boundary conditions on a fermionic field we have discussed above. Namely, one should take $\mu=m a-1 / 2, \delta=1$ for the condition (2.5), $\mu=m a+1 / 2, \quad \delta=-1$ for the condition (5.1), and $\mu=m a \pm 1 / 2, \delta=0$ for the remaining two boundary conditions discussed in Sec. VI. In [50,51], on the base of the generalized Abel-Plana formula, a summation formula is derived for the series over zeros of the 
function $\overline{\boldsymbol{J}}_{\mu}^{(a)}(x) \bar{Y}_{\mu}^{(b)}(\eta x)-\bar{Y}_{\mu}^{(a)}(x) \bar{J}_{\mu}^{(b)}(\eta x)$ with the notations $\bar{f}_{\mu}^{(j)}(z)=A_{j} f(z)+B_{j} z f^{\prime}(z)$, where $j=a, b$, and $A_{j}$, $B_{j}$ are constants. We take in that formula special values
$A_{b}=1, B_{b}=0, A_{a}=\mu^{|\delta|}, B_{a}=-\delta$. By using the recurrence relations for the modified Bessel functions the following formula is obtained

$$
\sum_{n=1}^{\infty} h\left(\lambda_{\mu, n}^{(\delta)}\right) T_{\mu}^{(\delta)}\left(\eta, \lambda_{\mu, n}^{(\delta)}\right)=\frac{2}{\pi^{2}} \int_{0}^{\infty} \frac{h(x) d x}{J_{\mu+\delta}^{2}(x)+Y_{\mu+\delta}^{2}(x)}+\frac{1}{2 \pi} \int_{0}^{\infty} d x \frac{\left[h\left(x e^{\pi i / 2}\right)+h\left(x e^{-\pi i / 2}\right)\right] K_{\mu}(\eta x) / K_{\mu+\delta}(x)}{I_{\mu+\delta}(x) K_{\mu}(\eta x)-(-1)^{\delta} K_{\mu+\delta}(x) I_{\mu}(\eta x)}
$$

where $h(z)$ is an analytic function in the right half-plane of the complex variable $z, I_{\mu}(x), K_{\mu}(x)$ are the modified Bessel functions and

$$
T_{\mu}^{(\delta)}(\eta, x)=\frac{x}{J_{\mu+\delta}^{2}(x) / J_{\mu}^{2}(\eta x)-1} .
$$

Note that the function in the denominator of the second integral in (A1) is equal to $G_{\mu+\delta, \mu}(x, \eta x)$ [see (4.9)].

The function $h(z)$ may have branch points on the imaginary axis that should be avoided by small semicircles in the right half-plane. Depending on the behavior of the function $h(z)$ near the origin, a residue term at $z=0$ may be present in the right-hand side of (A1) (see [51]). The corresponding contribution to the current density is cancelled by the contribution of the fermionic zero mode (for the case of a scalar field see [28]). By using the relation between the functions $I_{ \pm \mu}(x)$ and $K_{\mu}(x)$, it can bee seen that for $\delta=0$ one gets $G_{\mu, \mu}(x, \eta x)=G_{|\mu|,|\mu|}(x, \eta x)<0$. For $\delta= \pm 1$ and $\mu \geq 0$ one has $G_{\mu+\delta, \mu}(x, \eta x)>0$. In particular, from here it follows that for the boundary conditions we have discussed above and for $m a \geq 0$ there are no fermionic modes with purely imaginary $\lambda$.
[1] N. D. Birrell and P.C. W. Davies, Quantum Fields in Curved Space (Cambridge University Press, Cambridge, England, 1982); A. A. Grib, S. G. Mamayev, and V. M. Mostepanenko, Vacuum Quantum Effects in Strong Fields (Friedmann Laboratory Publishing, St. Petersburg, 1994); L. E. Parker and D. J. Toms, Quantum Field Theory in Curved Spacetime (Cambridge University Press, Cambridge, England, 2009).

[2] V. M. Mostepanenko and N. N. Trunov, The Casimir Effect and Its Applications (Oxford University Press, Oxford, 1997); K. A. Milton, The Casimir Effect: Physical Manifestation of Zero-Point Energy (World Scientific, Singapore, 2002); M. Bordag, G. L. Klimchitskaya, U. Mohideen, and V. M. Mostepanenko, Advances in the Casimir Effect (Oxford University Press, New York, 2009); Casimir Physics, edited by D. Dalvit, P. Milonni, D. Roberts, and F. da Rosa, Lecture Notes in Physics Vol. 834 (SpringerVerlag, Berlin, 2011).

[3] R. Maartens and K. Koyama, Living Rev. Relativity 13, 5 (2010).

[4] O. Aharony, S. S. Gubser, J. Maldacena, H. Ooguri, and Y. Oz, Phys. Rep. 323, 183 (2000); H. Năstase, Introduction to AdS/CFT Correspondence (Cambridge University Press, Cambridge, England, 2015); M. Ammon and J. Erdmenger, Gauge/Gravity Duality: Foundations and Applications (Cambridge University Press, Cambridge, England, 2015).

[5] A.S.T. Pires, AdS/CFT Correspondence in Condensed Matter (Morgan \& Claypool Publishers, USA, 2014); J. Zaanen, Y.-W. Sun, Y. Liu, and K. Schalm, Holographic Duality in Condensed Matter Physics (Cambridge
University Press, Cambridge, England, 2015); R.-G. Cai, L. Li, L.-F. Li, and R.-Q. Yang, Sci. China-Phys. Mech. Astron. 58, 060401 (2015); E. Kiritsis and L. Li, J. High Energy Phys. 01 (2016) 147.

[6] M. Fabinger and P. Horava, Nucl. Phys. B580, 243 (2000); S. Nojiri, S. Odintsov, and S. Zerbini, Phys. Rev. D 62, 064006 (2000); S. Nojiri, O. Obregon, and S. Odintsov, Phys. Rev. D 62, 104003 (2000); D. J. Toms, Phys. Lett. B 484, 149 (2000); W. D. Goldberger and I. Z. Rothstein, Phys. Lett. B 491, 339 (2000); S. Nojiri and S. Odintsov, J. High Energy Phys. 07 (2000) 049; J. Garriga, O. Pujolàs, and T. Tanaka, Nucl. Phys. B605, 192 (2001); I. H. Brevik, K. A. Milton, S. Nojiri, and S. D. Odintsov, Nucl. Phys. B599, 305 (2001); A. Flachi and D. J. Toms, Nucl. Phys. B610, 144 (2001); A. A. Saharian and M. R. Setare, Phys. Lett. B 552, 119 (2003); E. Elizalde, S. Nojiri, S. D. Odintsov, and S. Ogushi, Phys. Rev. D 67, 063515 (2003); J. Garriga and A. Pomarol, Phys. Lett. B 560, 91 (2003); R. A. Knapman and D. J. Toms, Phys. Rev. D 69, 044023 (2004); A. A. Saharian, Nucl. Phys. B712, 196 (2005); R. Durrer and M. Ruser, Phys. Rev. Lett. 99, 071601 (2007); M. Ruser and R. Durrer, Phys. Rev. D 76, 104014 (2007); A. A. Saharian and A. L. Mkhitaryan, J. High Energy Phys. 08 (2007) 063; M. Frank, I. Turan, and L. Ziegler, Phys. Rev. D 76, 015008 (2007); A. Flachi and T. Tanaka, Phys. Rev. D 80, 124022 (2009); L. P. Teo, Phys. Lett. B 682, 259 (2009); M. Rypestol and I. Brevik, New J. Phys. 12, 013022 (2010); R. Obousy and G. Cleaver, J. Geom. Phys. 61, 577 (2011); E. R. Bezerra de Mello, 
A. A. Saharian, and M. R. Setare, Phys. Rev. D 92, 104005 (2015); N. Haba and T. Yamada, arXiv:1903.10160.

[7] A. Flachi, I. G. Moss, and D. J. Toms, Phys. Lett. B 518, 153 (2001).

[8] A. Flachi, I. G. Moss, and D. J. Toms, Phys. Rev. D 64, 105029 (2001).

[9] S.-H. Shao, P. Chen, and J.-A. Gu, Phys. Rev. D 81, 084036 (2010).

[10] E. Elizalde, S. D. Odintsov, and A. A. Saharian, Phys. Rev. D 87, 084003 (2013); L. P. Teo, Int. J. Mod. Phys. A 28, 1350158 (2013).

[11] L. P. Teo, J. High Energy Phys. 10 (2010) 019; A. S. Kotanjyan and A. A. Saharian, Phys. At. Nucl. 80, 562 (2017); A. S. Kotanjyan, A. A. Saharian, H. G. Sargsyan, and D. H. Simonyan, Proc. Sci., MPCS2015 (2015) 021.

[12] S. Nojiri and S. Odintsov, Phys. Lett. B 484, 119 (2000); W. Naylor and M. Sasaki, Phys. Lett. B 542, 289 (2002); E. Elizalde, S. Nojiri, S. D. Odintsov, and S. Ogushi, Phys. Rev. D 67, 063515 (2003); I. G. Moss, W. Naylor, W. SantiagoGermán, and M. Sasaki, Phys. Rev. D 67, 125010 (2003); O. Pujolàs and T. Tanaka, J. Cosmol. Astropart. Phys. 12 (2004) 009; A. Flachi, A. Knapman, W. Naylor, and M. Sasaki, Phys. Rev. D 70, 124011 (2004); W. Naylor and M. Sasaki, Prog. Theor. Phys. 113, 535 (2005); O. Pujolàs and M. Sasaki, J. Cosmol. Astropart. Phys. 09 (2005) 002.

[13] A. Flachi, J. Garriga, O. Pujolàs, and T. Tanaka, J. High Energy Phys. 08 (2003) 053; A. Flachi and O. Pujolàs, Phys. Rev. D 68, 025023 (2003); A. A. Saharian, Phys. Rev. D 73, 044012 (2006); 73, 064019 (2006); 74, 124009 (2006); E. Elizalde, M. Minamitsuji, and W. Naylor, Phys. Rev. D 75, 064032 (2007); R. Linares, H. A. Morales-Técotl, and O. Pedraza, Phys. Rev. D 77, 066012 (2008); M. Frank, N. Saad, and I. Turan, Phys. Rev. D 78, 055014 (2008).

[14] A. A. Saharian, Phys. Rev. D 70, 064026 (2004); 74, 124009 (2006); A. A. Saharian and H. G. Sargsyan, Astrophysics 61, 375 (2018).

[15] E. R. Bezerra de Mello and A. A. Saharian, Phys. Rev. D 87, 045015 (2013).

[16] S. Bellucci, A. A. Saharian, and V. M. Bardeghyan, Phys. Rev. D 82, 065011 (2010).

[17] S. Bellucci, E. R. Bezerra de Mello, and A. A. Saharian, Phys. Rev. D 89, 085002 (2014).

[18] S. Bellucci and A. A. Saharian, Phys. Rev. D 87, 025005 (2013).

[19] S. Bellucci, A. A. Saharian, and N. A. Saharyan, Eur. Phys. J. C 75, 378 (2015).

[20] P. Recher, B. Trauzettel, A. Rycerz, Y. M. Blanter, C. W. J. Beenakker, and A. F. Morpurgo, Phys. Rev. B 76, 235404 (2007); S. Bellucci, A. A. Saharian, and A. Kh. Grigoryan, Phys. Rev. D 94, 105007 (2016).

[21] H. Bluhm, N. Koshnick, J. Bert, M. Huber, and K. Moler, Phys. Rev. Lett. 102, 136802 (2009); A. C. BleszynskiJayich, W. E. Shanks, B. Peaudecerf, E. Ginossar, F. von Oppen, L. Glazman, and J. G. E. Harris, Science 326, 272 (2009).

[22] E. R. Bezerra de Mello, V. Bezerra, A. A. Saharian, and V. M. Bardeghyan, Phys. Rev. D 82, 085033 (2010); S. Bellucci, E. R. Bezerra de Mello, and A. A. Saharian, Phys. Rev. D 83, 085017 (2011); A. A. Saharian, E. R. Bezerra de Mello, and A. A. Saharyan, Phys. Rev. D 100, 105014 (2019).
[23] S. Bellucci, A. A. Saharian, and H. A. Nersisyan, Phys. Rev. D 88, 024028 (2013).

[24] E. R. Bezerra de Mello, A. A. Saharian, and V. Vardanyan, Phys. Lett. B 741, 155 (2015).

[25] S. Bellucci, A. A. Saharian, and V. Vardanyan, Phys. Rev. D 96, 065025 (2017).

[26] W. Oliveira dos Santos, H. F. Mota, and E. R. Bezerra de Mello, Phys. Rev. D 99, 045005 (2019).

[27] S. Bellucci, A. A. Saharian, and V. Vardanyan, J. High Energy Phys. 11 (2015) 092.

[28] S. Bellucci, A. A. Saharian, and V. Vardanyan, Phys. Rev. D 93, 084011 (2016).

[29] S. Bellucci, A. A. Saharian, D. H. Simonyan, and V. Vardanyan, Phys. Rev. D 98, 085020 (2018).

[30] A. P. Prudnikov, Yu. A. Brychkov, and O. I. Marichev, Integrals and Series (Gordon and Breach, New York, 1986), Vol. 2.

[31] V.E. Ambruş and E. Winstanley, Phys. Lett. B 749, 597 (2015); Classical Quantum Gravity 34, 145010 (2017).

[32] Handbook of Mathematical Functions, edited by M. Abramowitz and I. A. Stegun (Dover, New York, 1972).

[33] M. V. Berry and R. J. Mondragon, Proc. R. Soc. A 412, 53 (1987).

[34] E. McCann and V. I. Fal'ko, J. Phys. Condens. Matter 16, 2371 (2004); L. Brey and H. A. Fertig, Phys. Rev. B 73, 235411 (2006); A. R. Akhmerov and C. W. J. Beenakker, Phys. Rev. B 77, 085423 (2008).

[35] M. H. Al-Hashimi and U.-J. Wiese, Ann. Phys. (Amsterdam) 327, 1 (2012); Yu. A. Sitenko, Phys. Rev. D 91, 085012 (2015).

[36] L. Randall and R. Sundrum, Phys. Rev. Lett. 83, 3370 (1999); 83, 4690 (1999).

[37] W. D. Goldberger and M. B. Wise, Phys. Rev. Lett. 83, 4922 (1999).

[38] S. Chan, S. Ch. Park, and J. Song, Phys. Rev. D 71, 106004 (2005).

[39] Y. Hosotani, Phys. Lett. B 126, 309 (1983); D. J. Toms, Phys. Lett. 126B, 445 (1983); Y. Hosotani, Ann. Phys. (N.Y.) 190, 233 (1989).

[40] H. Hatanaka, T. Inami, and C. S. Lim, Mod. Phys. Lett. A 13, 2601 (1998); M. Kubo, C. S. Lim, and H. Yamashita, Mod. Phys. Lett. A 17, 2249 (2002); N. Maru and K. Takenaga, Phys. Lett. B 637, 287 (2006); M. Sakamoto and K. Takenaga, Phys. Rev. D 80, 085016 (2009); K. Kashiwa and T. Misumi, J. High Energy Phys. 05 (2013) 042.

[41] V. P. Gusynin, S. G. Sharapov, and J. P. Carbotte, Int. J. Mod. Phys. B 21, 4611 (2007); A. H. Castro Neto, F. Guinea, N. M. R. Peres, K. S. Novoselov, and A. K. Geim, Rev. Mod. Phys. 81, 109 (2009).

[42] The Role of Topology in Materials, edited by S. Gupta and A. Saxena (Springer, Switzerland, 2018).

[43] D. V. Kolesnikov and V. A. Osipov, Phys. Part. Nucl. 40, 502 (2009); M. A. H. Vozmediano, M. I. Katsnelson, and F. Guinea, Phys. Rep. 496, 109 (2010).

[44] A. Iorio and G. Lambiase, Phys. Rev. D 90, 025006 (2014).

[45] T. Morresi, D. Binosi, S. Simonucci, R. Piergallini, S. Roche, N. M. Pugno, and S. Taioli, arXiv:1907.08960.

[46] J. González and J. Herrero, Nucl. Phys. B825, 426 (2010); V. Atanasov and A. Saxena, J. Phys. Condens. Matter 23, 
175301 (2011); R. Pincak and J. Smotlacha, Eur. Phys. J. B 86, 480 (2013); A. Sepehri, R. Pincak, K. Bamba, S. Capozziello, and E. N. Saridakis, Int. J. Mod. Phys. D 26, 1750094 (2017).

[47] G. E. Volovik and M. A. Zubkov, Ann. Phys. (Amsterdam) 356, 255 (2015); B. Amorim et al., Phys. Rep. 617, 1 (2016).

[48] T. Inagaki, T. Muta, and S. D. Odintsov, Prog. Theor. Phys. Suppl. 127, 93 (1997); A. Flachi, Phys. Rev. D 86, 104047 (2012); 88, 085011 (2013); A. Flachi, M. Nitta, S. Takada, and R. Yoshii, Phys. Rev. Lett. 119, 031601 (2017); A. Flachi and V. Vitagliano, Phys. Rev. D 99, 125010 (2019).
[49] A. Cortijo, F. Guinea, and M. A. H. Vozmediano, J. Phys. A 45, 383001 (2012); V. N. Kotov, B. Uchoa, V. M. Pereira, F. Guinea, and A. H. Castro Neto, Rev. Mod. Phys. 84, 1067 (2012).

[50] A. A. Saharian, Izvestiia Akademii nauk Armianskoi SSR Matematika 22, 166 (1987) [English translation: Sov. J. Contemp. Math. Anal. 22, 70 (1987)].

[51] A. A. Saharian, The Generalized Abel-Plana Formula with Applications to Bessel Functions and Casimir Effect (Yerevan State University Publishing House, Yerevan, 2008); Report No. ICTP/2007/082; arXiv:0708.1187. 